\title{
Finite-Time Projective Synchronization of Fractional-Order Memristive Neural Networks with Mixed Time-Varying Delays
}

\author{
Meng Hui $\mathbb{D}^{1},{ }^{1}$ Chen Wei $\left(\mathbb{D},{ }^{1}\right.$ Jiao Zhang, ${ }^{2}$ Herbert Ho-Ching Iu $\left(\mathbb{D},{ }^{3}\right.$ Ni Luo, ${ }^{1}$ Rui Yao, ${ }^{1}$ \\ and Lin Bai ${ }^{1}$ \\ ${ }^{1}$ School of Electronic and Control, Chang'an University, Xi'an, Shaanxi 710064, China \\ ${ }^{2}$ School of Electrical Engineering, Xi'an Jiaotong University, Xi'an, Shaanxi 710049, China \\ ${ }^{3}$ School of Electrical, Electronic and Computer Engineering, University of Western Australia, Perth, WA 6009, Australia \\ Correspondence should be addressed to Chen Wei; 2018132044@chd.edu.cn
}

Received 9 March 2020; Revised 9 May 2020; Accepted 27 May 2020; Published 27 June 2020

Academic Editor: Marcio Eisencraft

Copyright (c) 2020 Meng Hui et al. This is an open access article distributed under the Creative Commons Attribution License, which permits unrestricted use, distribution, and reproduction in any medium, provided the original work is properly cited.

This paper is concerned with the finite-time projective synchronization problem of fractional-order memristive neural networks (FMNNs) with mixed time-varying delays. Firstly, under the frame of fractional-order differential inclusion and the set-valued map, several criteria are derived to ensure finite-time projective synchronization of FMNNs. Meanwhile, three properties are established to deal with different forms of the finite-time fractional differential inequation, which greatly extend some results on estimation of settling time of FMNNs. In addition to the traditional Lyapunov function with 1-norm form in Theorem 1, a more general and flexible Lyapunov function based on p-norm is constructed in Theorem 2 to analyze the finite-time projective synchronization problem, and the estimation of settling time has been verified less conservative than previous results. Finally, numerical examples are provided to demonstrate the effectiveness of the derived theoretical results.

\section{Introduction}

In the past several decades, artificial neural networks have been extensively investigated due to their wide applications in signal processing [1], combinatorial optimization [2], pattern recognition [3], associative memories [4], and so on. Memristor, first proposed by Chua in 1971 [5], was realized by Hewlett-Packard Laboratory in 2008 [6]. Compared with the resistor, the memristor possesses a memory characteristic as the synaptic characteristics of biological neurons, which is distinct from other circuit elements. As a result, by using the memristor to replace other resistors, scholars constructed the memristive neural networks (MNNs) to emulate the human brain. Moreover, as a special kind of neural networks, MNNs are nonlinear systems with statedependent switching jumps and possess more complicated properties than the traditional neural networks.

It is well known that time delays are unavoidable in neural networks. On the one hand, discrete time delays are ubiquitous because of the finite switching speed of amplifiers and the inherent information exchanging time between different neurons [7]. On the other hand, distributed time delays should also be considered in neural networks because neural networks usually have a spatial nature and the presence of a large number of parallel pathways with multifarious axon sizes and lengths [8]. Hence, these time delays, which may bring about instability, chaos, oscillation, or other poor performances of the system, should be considered when investigating the dynamical behavior for neural networks [9-11].

As a generalization of the ordinary differentiation and integration to arbitrary noninteger order, fractional-order calculus has long history and can be traced back to the 17th century. For a long time, the theory of fractional calculus was once considered as a purely theoretical field of mathematics and developed very slow. However, due to its infinite memory and more degrees of freedom [12], many scholars have found that fractional-order models are more accurate than integer-order ones in describing the memory and hereditary properties. In consequence, fractional-order 
models have been incorporated into artificial neural networks. Subsequently, fractional-order memristive neural networks (FMNNs) have attracted lots of researchers to study their dynamical behaviors [13-15].

Since chaos synchronization was firstly proposed in 1990 [16], synchronization control has become an important tool to control the chaos appearing in the practical applications such as security communications [17], image processing [18], and cryptography [19]. So far, many sorts of synchronization schemes have been presented such as complete synchronization [20], antisynchronization [21], phase synchronization [22], lag synchronization [23], exponential synchronization [24], and projective synchronization.

Overall, the above discussed synchronization schemes can be divided into two categories: finite-time and infinitetime synchronization. In practice, the system always expected to realize synchronization as quickly as possible. It is worth noting that the finite-time control technique can not only accelerate the convergence process but also demonstrate better interference suppression properties and robustness [25]. Consequently, the finite-time synchronization is an optimal synchronization method and has attracted lots of attention in many research studies [26-33]. For example, in [27], by using the Gronwall-Bellman integral inequality and the Volterra integral equation, the authors explored the finite-time projective synchronization problem of memristor-based delay fractional-order neural networks. And Zhang and Deng studied the finite-time projective synchronization of fractional-order complex-valued memristor-based neural networks with time delays in [28]. However, we note that [26-28] pay attention to discussing the synchronization conditions and ignore the computation of settling time. In [29], Zhang et al. deduced the fractionalorder derivative of Lyapunov function ${ }_{t_{0}}^{C} D_{t}^{\alpha} V(t) \leq-a V(t)-$ $b$ and gave a conservative estimation of settling time with $a=0$. In [30-33], the authors deduced the fractional-order derivative of Lyapunov function ${ }_{t_{0}}^{C} D_{t}^{\alpha} V(t) \leq-$ $a V(t)-b V^{\beta}(t)$ and gave a conservative estimation of settling time with $a=0$. And we propose a new method to estimate settling time with $a \geq 0$, which is less conservative than previous results.

Among a great variety of synchronization schemes, projective synchronization, first proposed by Mainieri and Rehacek in [34], is characterized by a fact that the drive and response systems could achieve synchronization up to a scaling factor. Particularly, due to the proportional feature, projective synchronization can be used to extend binary digital to M-nary digital communication for achieving fast communication [35]. Furthermore, projective synchronization of FMNNs, which can obtain faster communication with its proportional feature, has been widely concerned and deeply studied by researchers [36-40]. For instance, the global projective synchronization for FMNNs is investigated via combining open-loop control with the time-delayed feedback control in [36]. And in [38], Yang et al. discussed the quasi-projective synchronization of fractional-order complex-valued neural networks, and the error bounds of quasi-projective synchronization are estimated. Based on notoriously Barbalat's lemma and the Razumikhin-type stability theorem, Zhang et al. investigated the projective synchronization of FMNNs with switching jump mismatch in [40]. However, to the best of our knowledge, there are few research studies about the finite-time projective synchronization of FMNNs with mixed time-varying delays.

Inspired by the above discussion, this paper will study the finite-time projective synchronization of FMNNs with mixed time-varying delays via applying differential inequality of the Caputo derivative and the asymptotic expansion property of Mittag-Leffler function. The main innovations and contents of this paper lie in the following aspects:

(1) For fractional-order neural networks, the fractionalorder derivative of Lyapunov function $V(t)$ can usually be reduced to ${ }_{t_{0}}^{C} D_{t}^{\alpha} V(t) \leq-a V(t)-b$ or ${ }_{t_{0}}^{C} D_{t}^{\alpha} V(t) \leq-a V(t)-b V^{\beta}(t)$. In the existing literature studies on finite-time synchronization of fractional-order neural networks, most pay attention to discuss the synchronization condition $a \geq 0$ and ignore the computation of settling time. And a few literature studies estimate the settling time with $a=0$, which will increase the conservative of the obtained result. In this paper, via applying the asymptotic expansion property of Mittag-Leffler function and the fractional-order power law inequation, we propose three properties to estimate the settling time with $a \geq 0$. And the comparison result can be seen in Tables 1-3, which demonstrate our new estimation of the settling time is more applicative and accurate.

(2) First, via designing a simple time-delayed feedback controller, some sufficient conditions are derived to guarantee the finite-time projective synchronization of FMNNs. And the results can be easily extended to the complete synchronization and antisynchronization.

(3) Unlike the traditional maximum absolute valuebased method to propose the synaptic weights of memristive neural networks, by introducing some transformations, FMNNs are translated to a type of fractional-order systems with uncertain parameters.

(4) In addition to the traditional Lyapunov function with 1-norm form, a more general and flexible Lyapunov function based on p-norm is constructed to analyze the projective finite-time synchronization of FMNNs.

The rest of this paper is organized as follows. Section 2 describes the models and introduces the preliminaries including some necessary definitions and lemmas. In Section 3 , we present the main results. Simulation examples are given to confirm the validity of our results in Section 4. And conclusions follow in Section 5.

Notation: throughout this study, $\mathbb{N}, \mathbb{C}, \mathbb{R}$, and $\mathbb{R}^{*}$ denote the set of all natural numbers, complex numbers, real numbers, and nonnegative real numbers, respectively. $C^{n}\left(\left[t_{0}, \infty\right), \mathbb{R}\right)$ represents the family of continuous and $n$ order differentiable function form $\left[t_{0}, \infty\right)$ to $\mathbb{R}$. And $\operatorname{sign}(\cdot)$ 
TABle 1: The comparisons among $T_{1}^{*}, T_{2}, T_{3}^{*}, T_{4}$, and $T_{5}$ with the projective coefficient $\lambda=2$.

\begin{tabular}{lccc}
\hline & Lyapunov function & Fractional-order differential inequation & Settling time \\
\hline Theorem 1 & ${ }_{t_{0}}^{C} D_{t}^{\alpha} V_{1}(t) \leq-\varphi V_{1}(t)-\eta$ & $T_{1}^{*}=1.235$ \\
Remark 4 & ${ }_{t_{0}}^{C} D_{t}^{\alpha} V_{1}(t) \leq-\eta$ & $T_{2}=5.5778$ \\
\hline Theorem 2 & $\sum_{i=1}^{n}\left|e_{i}(t)\right|$ & ${ }_{t_{0}}^{C} D_{t}^{\alpha} V_{2}(t) \leq-\bar{\varphi} V_{2}(t)-\bar{\eta} V_{2}^{(p-1) / p}(t)$ & $T_{3}^{*}=1.9054$ \\
Remark 6 & ${ }_{t_{0}}^{C} D_{t}^{\alpha} V_{2}(t) \leq-\bar{\eta} V_{2}^{(p-1) / p}(t)$ & $T_{4}=8.0421$ \\
& & $T_{2}(t)=\sum_{i=1}^{n}\left|e_{i}(t)\right|^{p}$ & $T_{5}=6.689$ \\
\hline
\end{tabular}

TABLE 2: The comparisons among $T_{1}^{*}, T_{2}, T_{3}^{*}, T_{4}$, and $T_{5}$ with the projective coefficient $\lambda=1$.

\begin{tabular}{lccc}
\hline & Lyapunov function & Fractional-order differential inequation & Settling time \\
\hline Theorem 1 & ${ }_{{ }_{0}}^{C} D_{t}^{\alpha} V_{1}(t) \leq-\varphi V_{1}(t)-\eta$ & $T_{1}^{*}=1.0351$ \\
Remark 4 & ${ }^{C}{ }_{1}(t)=\sum_{i=1}^{n}\left|e_{i}(t)\right|$ & $T_{t}^{\alpha} V_{1}(t) \leq-\eta$ & $T_{2}=3.5169$ \\
\hline Theorem 2 & ${ }_{t_{0}} D_{t}^{\alpha} V_{2}(t) \leq-\bar{\varphi} V_{2}(t)-\bar{\eta} V_{2}^{(p-1) / p}(t)$ & $T_{3}^{*}=1.5937$ \\
Remark 6 & ${ }_{t_{0}}^{C} D_{t}^{\alpha} V_{2}(t) \leq-\bar{\eta} V_{2}^{(p-1) / p}(t)$ & $T_{4}=5.1232$ \\
& & $V_{2}(t)=\sum_{i=1}^{n}\left|e_{i}(t)\right|^{p}$ & $T_{5}=4.4965$ \\
\hline
\end{tabular}

TABLE 3: The comparisons among $T_{1}^{*}, T_{2}, T_{3}^{*}, T_{4}$, and $T_{5}$ with the projective coefficient $\lambda=-1$.

\begin{tabular}{lccc}
\hline & Lyapunov function & Fractional-order differential inequation & Settling time \\
\hline Theorem 1 & ${ }_{t_{0}}^{C} D_{t}^{\alpha} V_{1}(t) \leq-\varphi V_{1}(t)-\eta$ & $T_{1}^{*}=0.6934$ \\
Remark 4 & ${ }_{{ }_{t}} D_{t}^{\alpha} V_{1}(t) \leq-\eta$ & $T_{2}=1.4814$ \\
\hline Theorem 2 & $V_{1}(t)=\sum_{i=1}^{n}\left|e_{i}(t)\right|$ & ${ }_{t_{0}}^{C} D_{t}^{\alpha} V_{2}(t) \leq-\bar{\varphi} V_{2}(t)-\bar{\eta} V_{2}^{(p-1) / p}(t)$ & $T_{3}^{*}=1.3539$ \\
Remark 6 & ${ }_{t_{0}}^{C} D_{t}^{\alpha} V_{2}(t) \leq-\bar{\eta} V_{2}^{(p-1) / p}(t)$ & $T_{4}=2.3539$ \\
& $V_{2}(t)=\sum_{i=1}^{n}\left|e_{i}(t)\right|^{p}$ & $T_{5}=2.1816$ \\
\hline
\end{tabular}

denotes the sign function, where sign $(x)=\left\{\begin{array}{ll}1, & \text { if } x \geq 0 \\ -1, & \text { if } x<0\end{array}\right.$. The symbol * involves the convolution operator. And $\Gamma(\alpha)$ is Euler's gamma function which is defined by $\Gamma(\alpha)=\int_{0}^{+\infty} s^{\alpha-1} e^{-s} \mathrm{~d} s$.

\section{Preliminaries and Model Description}

2.1. Preliminaries. In this section, we recall some basic definitions with respect to fractional calculus and several important lemmas used in this paper.

Definition 1 (see [41]). The Riemann-Liouville fractional integral with fractional-order $\alpha$ for an integrable function $f(t)$ is defined as

$$
{ }_{t_{0}} I_{t}^{\alpha} f(t)=\frac{1}{\Gamma(\alpha)} \int_{t_{0}}^{t}(t-s)^{\alpha-1} f(s) \mathrm{d} s
$$

where $t \geq t_{0}$ and $\alpha>0$.

Definition 2 (see [42]). The Caputo fractional derivative with fractional-order $\alpha$ for a differentiable function $f(t) \in C^{n}\left(\left[t_{0},+\infty\right), \mathbb{R}\right)$ is defined as

$$
{ }_{t_{0}}^{C} D_{t}^{\alpha} f(t)=\frac{1}{\Gamma(n-\alpha)} \int_{t_{0}}^{t} \frac{f^{(n)}(s)}{(t-s)^{\alpha-n+1}} \mathrm{~d} s,
$$

where $t \geq t_{0}$ and $n$ is a positive integral such that $n-1<\alpha<n$. Especially, when $\alpha \in(0,1)$, then

$$
{ }_{t_{0}}^{C} D_{t}^{\alpha} f(t)=\frac{1}{\Gamma(1-\alpha)} \int_{t_{0}}^{t} \frac{f^{\prime}(s)}{(t-s)^{\alpha}} \mathrm{d} s .
$$

Definition 3 (see [41]). Mittag--Leffler function $E_{p, q}(\cdot)$ with two parameters is defined as

$$
E_{p, q}(\varsigma)=\sum_{k=0}^{\infty} \frac{\varsigma^{k}}{\Gamma(p k+q)}
$$

where $p>0, q>0$, and $\varsigma \in \mathbb{C}$. And if $q=1$, the Mittag-Leffler function with one parameter is shown as

$$
E_{p}(\varsigma)=E_{p, 1}(\varsigma)=\sum_{k=0}^{\infty} \frac{\varsigma^{k}}{\Gamma(p k+1)} \text {. }
$$

Definition 4. If there exists a nonzero constant $\lambda \in \mathbb{R}$, for any solutions of the $n$-dimensional drive system and the response system, $x_{i}(t)$ and $y_{i}(t), i=1,2, \ldots, n$, with initial values $x_{i}\left(t_{0}\right)$ and $y_{i}\left(t_{0}\right)$ are such that

$$
\begin{gathered}
\lim _{t \longrightarrow T^{*}}\left[y_{i}(t)-\lambda x_{i}(t)\right]=0, \\
y_{i}(t)-\lambda x_{i}(t) \equiv 0, \quad t \geq T^{*},
\end{gathered}
$$

where $\lambda$ represents the projective coefficient. $T^{*}$ is called the settling time for synchronization. Then, the response system is said to be finite-time projective synchronization to the drive system. 
Particularly, the drive-response system can be called finite-time complete synchronization if $\lambda=1$ and finite-time antisynchronization if $\lambda=-1$.

Lemma 1 (see [41]). If $f(t):\left[t_{0},+\infty\right) \longrightarrow \mathbb{R}$, then for all $t \in\left[t_{0},+\infty\right)$,

$$
t_{0} I_{t}^{\alpha}\left({ }_{t_{0}}^{C} D_{t}^{\alpha} f(t)\right)=f(t)-\sum_{i=1}^{n-1} \frac{f^{(i)}\left(t_{0}\right)}{i !}\left(t-t_{0}\right)^{i} .
$$

In particular, when $0<\alpha<1$, it has

$$
{ }_{t_{0}} I_{t}^{\alpha}\left({ }_{t_{0}}^{C} D_{t}^{\alpha} f(t)\right)=f(t)-f\left(t_{0}\right) \text {. }
$$

Lemma 2 (see [43]). For all $0<\alpha<2, \beta \in \mathbb{C}, m \in \mathbb{N}$, and $z \neq 0, \quad|\arg z|<\min \{\pi, \alpha \pi\}$, then the following asymptotic formula holds:

$$
E_{\alpha, \beta}(z)=\frac{1}{\alpha} z^{(1-\beta) \alpha} e^{z^{1 \alpha}}-\sum_{k=1}^{m} \frac{z^{-k}}{\Gamma(\beta-k \alpha)}+O\left[\frac{1}{z^{m+1}}\right], \quad|z| \longrightarrow \infty .
$$

Lemma 3 (see [44]). Let $z_{1}>0, z_{2}>0, z_{3}>1, z_{4}>1$, and $z_{3}^{-1}+z_{4}^{-1}=1$; then, the following inequality holds:

$$
z_{1} z_{2} \leq \varepsilon \frac{z_{1}^{z_{3}}}{z_{3}}+\varepsilon^{-z_{3} / z_{4}} \frac{z_{2}^{z_{4}}}{z_{4}}
$$

where $\varepsilon$ is an arbitrary positive constant.

Lemma 4 (see [45]). Assume that $a_{1}, a_{2}, \ldots, a_{n}$ are nonnegative constants. For two arbitrary constants $p$ and $q$ satisfying $0<p<q$, then

$$
\left(\sum_{i=1}^{n} a_{i}^{q}\right)^{1 / q} \leq\left(\sum_{i=1}^{n} a_{i}^{p}\right)^{1 / p}
$$

Lemma 5 (see [46]). Let $f(t)$ be a continuous function on the positive interval $\left[t_{0},+\infty\right)$ and satisfy

$$
{ }_{t_{0}}^{C} D_{t}^{\alpha} f(t) \leq a f(t)
$$

where $a$ is a constant and $0<\alpha<1$; then,

$$
f(t) \leq f\left(t_{0}\right) E_{\alpha}\left(a\left(t-t_{0}\right)^{\alpha}\right) .
$$

Lemma 6. Let $f(t)$ be a continuous function on the positive interval $\left[t_{0},+\infty\right)$ and satisfy

$$
{ }_{t_{0}}^{C} D_{t}^{\alpha} f(t) \leq a f(t)+b,
$$

where $a$ and $b$ are constants and $0<\alpha<1$; then,

$$
f(t) \leq\left(f\left(t_{0}\right)+\frac{b}{a}\right) E_{\alpha}\left(a\left(t-t_{0}\right)^{\alpha}\right)-\frac{b}{a} .
$$

Proof. Let $h(t)=f(t)+(b / a)$, and we can easily obtain

$$
{ }_{t_{0}}^{C} D_{t}^{\alpha} h(t)={ }_{t_{0}}^{C} D_{t}^{\alpha}(t) \leq a f(t)+b=a h(t) .
$$

By applying Lemma 5, we have $h(t) \leq$ $h\left(t_{0}\right) E_{\alpha}\left(a\left(t-t_{0}\right)^{\alpha}\right)$, which is equivalent to

$$
f(t) \leq\left(f\left(t_{0}\right)+\frac{b}{a}\right) E_{\alpha}\left(a\left(t-t_{0}\right)^{\alpha}\right)-\frac{b}{a} .
$$

Remark 1. For Lemma 6, Yang et al. in [38] obtain a consistent result with $a>0$ and $b \geq 0$. And we believe that the conditions of $a>0$ and $b \geq 0$ are conservative. And in Lemma 6 , we consider that $a$ and $b$ are constants, which is more general than the results in [38].

Lemma 7 (see [30]). Suppose $0<\alpha \leq 1, \quad \beta \geq 1$, and $f(t) \in C^{1}\left(\left[t_{0},+\infty\right), \mathbb{R}\right)$; then,

$$
{ }_{t_{0}}^{C} D_{t}^{\alpha} f^{\beta}(t)=\frac{\Gamma(2-\alpha) \Gamma(1+\beta)}{\Gamma(1+\beta-\alpha)} f^{\beta-1}(t){ }_{t_{0}}^{C} D_{t}^{\alpha} f(t) .
$$

Lemma 8 (see [47]). Let $f(t) \in C^{1}\left(\left[t_{0},+\infty\right), \mathbb{R}\right)$ be a continuous and differential function; the following inequation satisfies almost everywhere

$$
{ }_{t_{0}}^{C} D_{t}^{\alpha}|f(t)| \leq \operatorname{sign}(f(t))_{t_{0}}^{C} D_{t}^{\alpha} f(t),
$$

where $0<\alpha<1$.

In the following, two new fractional-order inequalities are established to propose the finite-time convergence, which play a critical role to obtain the finite-time synchronization and calculate the settling time. And we can gain three new properties.

Property 1. Let $f(t) \in C^{1}\left(\left[t_{0},+\infty\right), \mathbb{R}^{*}\right)$ be continuous and nonnegative definite. And the following inequation holds:

$$
{ }_{t_{0}}^{C} D_{t}^{\alpha} f(t) \leq-a f(t)-b
$$

where $a>0, b>0$, and $0<\alpha<1$. It can be deduced as follows:

$$
f(t) \leq\left[f\left(t_{0}\right)+\frac{b}{a}\right] \frac{1}{\alpha} \exp \left\{-a^{1 / \alpha}\left(t-t_{0}\right)\right\}-\frac{b}{a}, \quad t \geq t_{0} .
$$

Then, we can judge $f(t)$ converges to 0 within the settling time $T_{1}^{*}$ which can be estimated as follows:

$$
t_{1}^{*} \leq t_{0}+a^{-1 / \alpha} \ln \frac{a f\left(t_{0}\right)+b}{b \alpha} .
$$

Proof. By utilizing Lemma 6, we have

$$
f(t) \leq\left[f\left(t_{0}\right)+\frac{b}{a}\right] E_{\alpha}\left(-a\left(t-t_{0}\right)^{\alpha}\right)-\frac{b}{a} .
$$

From Lemma 2 , when $\beta=1$, we can get

$$
E_{\alpha}(z)=\frac{1}{\alpha} e^{z^{1 \alpha}}-\sum_{k=1}^{m} \frac{z^{-k}}{\Gamma(1-k \alpha)}+O\left[\frac{1}{z^{m+1}}\right], \quad|z| \longrightarrow \infty .
$$


Based on $\lim _{z \longrightarrow \infty} O\left[1 / z^{m+1}\right]=0$, we can easily gain

$$
\lim _{z \longrightarrow \infty} E_{\alpha}(z) \longrightarrow \lim _{z \longrightarrow \infty}\left[\frac{1}{\alpha} e^{z^{1 \alpha}}-\sum_{k=1}^{m} \frac{z^{-k}}{\Gamma(1-k \alpha)}\right]
$$

Apparently, $\sum_{k=1}^{m} z^{-k} / \Gamma(1-k \alpha)>0$; then, we can judge that there exists $z_{0}>0$ such that

$$
E_{\alpha}(z) \leq \frac{1}{\alpha} e^{z^{1 \alpha}}, \quad z>z_{0} .
$$

Similarly, we have

$$
E_{\alpha}\left(-a\left(t-t_{0}\right)^{\alpha}\right) \leq \frac{1}{\alpha} \exp \left\{-a^{1 / \alpha}\left(t-t_{0}\right)\right\}, \quad t \geq t_{0} .
$$

From the above conclusion, inequation (20) can be converted to

$$
f(t) \leq\left[f\left(t_{0}\right)+\frac{b}{a}\right] \frac{1}{\alpha} \exp \left\{-a^{1 / \alpha}\left(t-t_{0}\right)\right\}-\frac{b}{a}, \quad t \geq t_{0} .
$$

In order to better analyze the problem, let $\Omega(t)=$ $\left[f\left(t_{0}\right)+(b / a)\right](1 / \alpha) e^{-a^{1 / \alpha}\left(t-t_{0}\right)}-(b / a)$. We can easily know that the function $\Omega(t)$ is monotonically decreasing. And $\Omega\left(t_{0}\right)=(1 / \alpha) f\left(t_{0}\right)+((1 / \alpha)-1)(b / a)>(1 / \alpha) f\left(t_{0}\right)>0$ and $\lim _{t \rightarrow \infty} \Omega(t) \longrightarrow-(b / a)<0$. According to the zero-point theorem, there exists $t_{1}^{*} \in\left[t_{0}, \infty\right)$ such that $\Omega\left(t_{1}^{*}\right)=0$. Then, we can gain $0 \leq f\left(t_{1}^{*}\right) \leq \Omega\left(t_{1}^{*}\right)=0$, so we have $f\left(t_{1}^{*}\right)=0$. And when $t \geq t_{1}^{*}$, then $f(t) \equiv 0$.

Based on Definition 4, the function $f(t)$ converges to 0 in finite time. And the settling time $t_{1}^{*}$ can be calculated as follows:

$$
t_{1}^{*} \leq t_{0}+a^{-1 / \alpha} \ln \frac{a f\left(t_{0}\right)+b}{b \alpha} .
$$

Property 2. Assume that function $f(t) \in C\left(\left[t_{0},+\infty\right), \mathbb{R}^{*}\right)$ is continuous and nonnegative definite. If the following inequality holds,

$$
{ }_{t_{0}}^{C} D_{t}^{\alpha} f(t) \leq-a f^{\beta}(t)-b
$$

where $a>0, b>0,0<\alpha<1,0<\beta \leq 1$, then it can be deduced as follows:

$$
f(t) \leq\left[\left(f\left(t_{0}\right)+\left(\frac{b}{a}\right)^{1 / \beta}\right)^{1-\beta}-\frac{a \Gamma(1+(1 /(1-\beta))-\alpha)\left(t-t_{0}\right)^{\alpha}}{\Gamma(1+(1 /(1-\beta))) \Gamma(2-\alpha) \Gamma(1+\alpha)}\right]^{1 /(1-\beta)}-\left(\frac{b}{a}\right)^{1 / \beta}
$$

And we can judge $f(t)$ converges to 0 within the settling time $T_{2}^{*}$ which can be estimated as follows:

$$
t_{2}^{*} \leq t_{0}+\left[f^{1-\beta}\left(t_{0}\right) \frac{\Gamma(1+(1 /(1-\beta))) \Gamma(2-\alpha) \Gamma(1+\alpha)}{a \Gamma(1+(1 /(1-\beta))-\alpha)}\right]^{1 / \alpha} .
$$

Proof. Let $g(t)=f(t)+(b / a)^{1 / \beta}>0$; according to Lemma 4, we gain

$$
g^{\beta}(t)=\left(f(t)+\left(\frac{b}{a}\right)^{1 / \beta}\right)^{\beta} \leq f^{\beta}(t)+\frac{b}{a} .
$$

Multiplying $-a$ on sides of the above inequation, then we can get

$$
-a f^{\beta}(t)-b \leq-a g^{\beta}(t)
$$

According to the conditions of Property 2,

${ }_{t_{0}}^{C} D_{t}^{\alpha} g(t)={ }_{t_{0}}^{C} D_{t}^{\alpha} f(t) \leq-a f^{\beta}(t)-b \leq-a g^{\beta}(t)$.

Let $w(t)=g^{\beta / m}(t)$, where $m$ is a positive integer. Then, the above inequation can be written as follows:

$$
{ }_{t_{0}}^{C} D_{t}^{\alpha} w^{m / \beta}(t) \leq-a w^{m}(t)
$$

When $m \geq \beta$, via applying Lemma 7 , we can gain $\frac{\Gamma(2-\alpha) \Gamma(1+(m / \beta))}{\Gamma(1+(m / \beta)-\alpha)} w^{(m / \beta)-1}(t) D_{t}^{\alpha} w(t) \leq-a w^{m}(t)$.

Then,

$$
w^{(m / \beta)-m-1}(t) D_{t}^{\alpha} w(t) \leq \frac{-a \Gamma(1+(m / \beta)-\alpha)}{\Gamma(2-\alpha) \Gamma(1+(m / \beta))} .
$$

Considering $m \geq(\beta /(1-\beta))$, thus $(m / \beta)-m-1 \geq 0$. Once again, we apply Lemma 7 into the above inequation. And we have

$$
D_{t}^{\alpha} w^{(m / \beta)-m}(t) \leq \frac{-a \Gamma(1+(m / \beta)-\alpha) \Gamma(1+(m / \beta)-m)}{\Gamma(1+(m / \beta)) \Gamma(1+(m / \beta)-m-\alpha)} .
$$

Combining Lemma 1, we take the fractional integral with fractional-order $\alpha$ from $t_{0}$ to $t$ on sides of in equation (3); we have

$$
w^{(m / \beta)-m}(t)-w^{(m / \beta)-m}\left(t_{0}\right) \leq \frac{-a \Gamma(1+(m / \beta)-\alpha) \Gamma(1+(m / \beta)-m)\left(t-t_{0}\right)^{\alpha}}{\Gamma(1+(m / \beta)) \Gamma(1+(m / \beta)-m-\alpha) \Gamma(1+\alpha)} .
$$


Substituting $w(t)=\left[f(t)+(b / a)^{1 / \beta}\right]^{\beta / m}$ into the above inequation, we can obtain

$$
f(t) \leq\left[\left(f\left(t_{0}\right)+\left(\frac{b}{a}\right)^{1 / \beta}\right)^{1-\beta}-\frac{a \Gamma(1+(m / \beta)-\alpha) \Gamma(1+(m / \beta)-m)\left(t-t_{0}\right)^{\alpha}}{\Gamma(1+(m / \beta)) \Gamma(1+(m / \beta)-m-\alpha) \Gamma(1+\alpha)}\right]^{1 /(1-\beta)}-\left(\frac{b}{a}\right)^{1 / \beta} .
$$

Apparently, the nonnegative function $f(t)$ is monotonically decreasing. Hence, we can conclude that $f(t)$ converges to 0 within the settling time $t_{2}^{*}$. By simple computation, the estimation of settling time $t_{2}^{*}$ is given by

$$
t_{2}^{*} \leq t_{0}+\left[\left(\left(f\left(t_{0}\right)+\left(\frac{b}{a}\right)^{(1 / \beta)}\right)^{1-\beta}-\left(\frac{b}{a}\right)^{(1-\beta) / \beta}\right) \frac{\Gamma(1+(m / \beta)) \Gamma(1+(m / \beta)-m-\alpha) \Gamma(1+\alpha)}{a \Gamma(1+(m / \beta)-\alpha) \Gamma(1+(m / \beta)-m)}\right]^{1 / \alpha} .
$$

From the above analysis, the estimation of settling time is connected with a positive integer $m \geq(\beta /(1-\beta))$. Then, we would like to discuss the effect of $m$ for settling time. First, we define a function $H(m)$ as follows:

$$
H(m)=\frac{\Gamma(1+(m / \beta))}{\Gamma(1+(m / \beta)-m)} \cdot \frac{\Gamma(1+(m / \beta)-m-\alpha)}{\Gamma(1+(m / \beta)-\alpha)} .
$$

Based on the property of Euler's gamma function, we have

$$
H(m)=\frac{(1+(m / \beta)-1)(1+(m / \beta)-2) \cdots(1+(m / \beta)-m)}{(1+(m / \beta)-\alpha-1)(1+(m / \beta)-\alpha-2) \cdots(1+(m / \beta)-\alpha-m)}=\prod_{i=1}^{m} A_{i}
$$

where $A_{i}=(1+(m / \beta)-i) /(1+(m / \beta)-\alpha-i), i=1,2, \ldots$, $m$. Obviously, $A_{i}>1$. On the contrary,

$$
\frac{\mathrm{d} A_{i}}{\mathrm{~d} i}=\frac{\alpha}{(1+(m / \beta) \alpha-i)^{2}}>0 .
$$

According to the above analysis, it can be obtained that $H(m)$ is monotone increasing about $m$. Therefore, it can be deduced that the settling time is monotone increasing about $m$. In order to obtain more accurate estimation of settling time, we should choose a smaller value of $m$.

From the above analysis, if we consider $m=\beta /(1-\beta)$, we can gain a smaller estimation of settling time as follows:

$$
\begin{aligned}
t_{2}^{*} & \leq t_{0}+\left[\left(\left(f\left(t_{0}\right)+\left(\frac{b}{a}\right)^{1 / \beta}\right)^{1-\beta}-\left(\frac{b}{a}\right)^{(1-\beta) / \beta}\right) \frac{\Gamma(1+(1 /(1-\beta))) \Gamma(1+(1 /(1-\beta))-(\beta /(1-\beta))-\alpha) \Gamma(1+\alpha)}{a \Gamma(1+(1 /(1-\beta))-\alpha) \Gamma(1+(1 /(1-\beta))-(\beta /(1-\beta)))}\right]^{1 / \alpha} \\
& =t_{0}+\left[\left(\left(f\left(t_{0}\right)+\left(\frac{b}{a}\right)^{1 / \beta}\right)^{1-\beta}-\left(\frac{b}{a}\right)^{(1-\beta) / \beta}\right) \frac{\Gamma(1+(1 /(1-\beta))) \Gamma(2-\alpha) \Gamma(1+\alpha)}{a \Gamma(1+(1 /(1-\beta))-\alpha)}\right]^{1 / \alpha} .
\end{aligned}
$$

For Property 2, considering $b=0$, we can obtain Corollary 1 as follows.

Corollary 1. Assume that function $f(t) \in C\left(\left[t_{0},+\infty\right), \mathbb{R}^{*}\right)$ is continuous and nonnegative definite. If the following inequality holds,

$$
{ }_{t_{0}}^{C} D_{t}^{\alpha} f(t) \leq-a f^{\beta}(t)
$$

where $a>0,0<\alpha<1$, and $0<\beta \leq 1$, then it can be deduced as follows:

$$
f(t) \leq\left[f^{1-\beta}\left(t_{0}\right)-\frac{a \Gamma(1+(1 /(1-\beta))-\alpha)\left(t-t_{0}\right)^{\alpha}}{\Gamma(1+(1 /(1-\beta))) \Gamma(2-\alpha) \Gamma(1+\alpha)}\right]^{(1 /(1-\beta))} .
$$

And we can judge $f(t)$ converges to 0 within the settling time $t_{3}{ }^{*}$ which can be estimated as follows: 


$$
t_{3}^{*} \leq t_{0}+\left[f^{1-\beta}\left(t_{0}\right) \frac{\Gamma(1+(1 /(1-\beta))) \Gamma(2-\alpha) \Gamma(1+\alpha)}{a \Gamma(1+(1 /(1-\beta))-\alpha)}\right]^{1 / \alpha} .
$$

Remark 2. Peng et al. and $\mathrm{Wu}$ et al. in $[31,32]$ proposed the estimation of settling time for the differential inequation ${ }_{t_{0}}^{C} D_{t}^{\alpha} f(t) \leq-a f^{\beta}(t)$. According to the results in [31,32], we can get another estimation of settling time:

$$
t_{4}^{*} \leq t_{0}+\left[f^{\alpha(1+\alpha)-\beta-1}\left(t_{0}\right) \frac{\Gamma(1+\alpha) \Gamma(1-((1+\beta) /(1+\alpha)))}{a \Gamma(2+\alpha) \Gamma(1+\alpha-((1+\beta) /(1+\alpha)))}\right]^{1 / \alpha},
$$

where $0<\beta<\alpha(1+\alpha)-1$. Apparently, the results in [31,32] need a limiting condition on $\alpha$ and $\beta$. Compared with the above results, the estimation of settling time in Property 2 and Corollary 1 has no limits about $\alpha$ and $\beta$ when we select a suitable value of $m$. Hence, the result we obtained is more general and less conservative.

Property 3. Assume that function $f(t) \in C\left(\left[t_{0},+\infty\right), \mathbb{R}^{*}\right)$ is continuous and nonnegative definite. If the following inequality holds,

$$
{ }_{t_{0}}^{C} D_{t}^{\alpha} f(t) \leq-a f(t)-b f^{\beta}(t),
$$

where $a>0, b>0,0<\alpha<1$, and $0<\beta \leq 1$, then it can be deduced as follows:

$$
f^{1-\beta}(t) \leq\left[f^{1-\beta}\left(t_{0}\right)+\frac{b}{a}\right] \frac{1}{\alpha} \exp \left\{-\left(\frac{a \Gamma(1+(1 / \beta)-\alpha)}{\Gamma(1+(1 / \beta)) \Gamma(2-\alpha)}\right)^{1 / \alpha}\left(t-t_{0}\right)\right\}-\frac{b}{a}, \quad t \geq t_{0} .
$$

And we can judge $f(t)$ converges to 0 within the settling time $T_{2}^{*}$ which can be estimated as follows:

$$
t_{5}^{*} \leq t_{0}+\left[\frac{a \Gamma(1+(1 / \beta)-\alpha)}{\Gamma(1+(1 / \beta)) \Gamma(2-\alpha)}\right]^{-1 / \alpha} \ln \frac{a f^{1-\beta}\left(t_{0}\right)+b}{b \alpha} .
$$

Proof. Let $w(t)=f^{\beta / m}(t)$, where $m$ is a positive integer. Then, inequation (51) can be written as follows:

$$
{ }_{t_{0}}^{C} D_{t}^{\alpha} w^{m / \beta}(t) \leq-a w^{m / \beta}(t)-b w^{m}(t) .
$$

When $m \geq \beta$, via applying Lemma 7 , we can gain

$$
\frac{\Gamma(2-\alpha) \Gamma(1+(m / \beta))}{\Gamma(1+(m / \beta)-\alpha)} w^{(m / \beta)-1}(t) D_{t}^{\alpha} w(t) \leq-a w^{m / \beta}(t)-b w^{m}(t) .
$$

Then,

$$
\frac{\Gamma(2-\alpha) \Gamma(1+(m / \beta))}{\Gamma(1+(m / \beta)-\alpha)} w^{(m / \beta)-m-1}(t) D_{t}^{\alpha} w(t) \leq-a w^{(m / \beta)-m}(t)-b .
$$

Considering $m \geq(\beta /(1-\beta))$, thus $(m / \beta)-m-1 \geq 0$. Once again, we apply Lemma 7 into the above inequation. And we have

$$
D_{t}^{\alpha} w^{(m / \beta)-m}(t) \leq-\widehat{a} w^{(m / \beta)-m}(t)-\widehat{b},
$$

where

$$
\begin{aligned}
& \widehat{a}=\frac{\Gamma(1+(m / \beta)-\alpha) \Gamma(1+(m / \beta)-m)}{\Gamma(1+(m / \beta)) \Gamma(1+(m / \beta)-m-\alpha)} a, \\
& \widehat{b}=\frac{\Gamma(1+(m / \beta)-\alpha) \Gamma(1+(m / \beta)-m)}{\Gamma(1+(m / \beta)) \Gamma(1+(m / \beta)-m-\alpha)} b .
\end{aligned}
$$

Combining Property 1, inequation (57) can be written as follows:

$$
w^{(m / \beta)-m}(t) \leq\left[w^{(m / \beta)-m}\left(t_{0}\right)+\frac{\widehat{b}}{\widehat{a}}\right] \frac{1}{\alpha} \exp \left(-\widehat{a}^{1 / \alpha}\left(t-t_{0}\right)\right)-\frac{\widehat{b}}{\widehat{a}}, \quad t \geq t_{0} .
$$

Substituting $w(t)=f^{\beta / m}(t)$ and $\widehat{b} / \widehat{a}=b / a$ into the above inequation, we can obtain

$$
f^{1-\beta}(t) \leq\left[f^{1-\beta}\left(t_{0}\right)+\frac{b}{a}\right] \frac{1}{\alpha} \exp \left(-\widehat{a}^{1 / \alpha}\left(t-t_{0}\right)\right)-\frac{b}{a}, \quad t \geq t_{0} .
$$

Because $f(t)$ is a nonnegative function, we can gain $f(t)=0$ if $f^{1-\beta}(t)=0$. Therefore, based on the analysis in Property 1 , we can conclude that $f(t)$ converges to 0 within the settling time $t_{2}^{*}$. By simple computation, the estimation of settling time $t_{2}^{*}$ is given by

$$
t_{5}^{*} \leq t_{0}+\left[\frac{\Gamma(1+(m / \beta)-\alpha) \Gamma(1+(m / \beta)-m)}{(1+(m / \beta)) \Gamma(1+(m / \beta)-m-\alpha)} a\right]^{-1 / \alpha} \ln \frac{a f\left(t_{0}\right)+b}{b \alpha} .
$$

Similar to Property $1, H(m)=((\Gamma(1+(m / \beta))) /(\Gamma(1+$ $(m / \beta)-m))) \cdot((\Gamma(1+(m / \beta)-m-\alpha)) /(\Gamma(1+(m / \beta)-\alpha)))$ is monotone increasing about $m$.

$$
t_{5}^{*} \leq t_{0}+\left[\frac{a}{H(m)}\right]^{-1 / \alpha} \ln \frac{a f\left(t_{0}\right)+b}{b \alpha} .
$$

Therefore, it can be deduced that the settling time is monotone increasing about $m$. In order to obtain more accurate estimation of settling time, we should choose a smaller value of $\beta /(1-\beta)$. 


$$
f^{1-\beta}(t) \leq\left[f^{1-\beta}\left(t_{0}\right)+\frac{b}{a}\right] \frac{1}{\alpha} \exp \left\{-\left(\frac{a \Gamma(1+(1 / \beta)-\alpha)}{\Gamma(1+(1 / \beta)) \Gamma(2-\alpha)}\right)^{1 / \alpha}\left(t-t_{0}\right)\right\}-\frac{b}{a}, \quad t \geq t_{0} .
$$

And we can obtain the settling time $t_{2}{ }^{*}$ as follows:

$t_{5}^{*} \leq t_{0}+\left[\frac{a \Gamma(1+(1 / \beta)-\alpha)}{\Gamma(1+(1 / \beta)) \Gamma(2-\alpha)}\right]^{-1 / \alpha} \ln \frac{a f^{1-\beta}\left(t_{0}\right)+b}{b \alpha}$.

2.2. ModelDescription. A practical integer-order memristive neural network can usually be implemented by a very largescale integration (VISI) circuit as shown in Figure 1. According to Kirchhoff's current law, the ith subsystem with mixed delays can be described as follows:

$$
\begin{gathered}
\frac{\mathrm{d} x_{i}(t)}{\mathrm{d} t}=-d_{i} x_{i}(t)+\sum_{j=1}^{n} a_{i j}\left(x_{i}(t)\right) f_{j}\left(x_{j}(t)\right) \\
+\sum_{j=1}^{n} b_{i j}\left(x_{i}(t)\right) g_{j}\left(x_{j}\left(t-\tau_{j}(t)\right)\right) \\
+\sum_{j=1}^{n} c_{i j}\left(x_{i}(t)\right) \int_{t-\rho_{j}(t)}^{t} h_{j}\left(x_{j}(s)\right) \mathrm{d} s+I_{i}, \\
i=1,2, \ldots, n, t \geq t_{0} .
\end{gathered}
$$

In this paper, the above integer-order FNN model is expended to fractional-order FNNs to explore the finite-time projective synchronization. And the drive system can be described by the following differential equation:

$$
\begin{gathered}
{ }_{t_{0}}^{C} D_{t}^{\alpha} x_{i}(t)=-d_{i} x_{i}(t)+\sum_{j=1}^{n} a_{i j}\left(x_{i}(t)\right) f_{j}\left(x_{j}(t)\right) \\
+\sum_{j=1}^{n} b_{i j}\left(x_{i}(t)\right) g_{j}\left(x_{j}\left(t-\tau_{j}(t)\right)\right) \\
+\sum_{j=1}^{n} c_{i j}\left(x_{i}(t)\right) \int_{t-\rho_{j}(t)}^{t} h_{j}\left(x_{j}(s)\right) \mathrm{d} s+I_{i}, \\
i=1,2, \ldots, n, t \geq t_{0} .
\end{gathered}
$$

where ${ }_{t_{0}}^{C} D_{t}^{\alpha}$ denotes the Caputo fractional derivative of order $0<\alpha<1$ from $t_{0}$ to $t ; d_{i} \geq 0$ is the self-feedback connection weight of the $i$ th neuron; $x_{i}(t)$ represents the corresponding state variable of the number of $i$ th neurons at time $t ; f_{j}(\cdot)$, $g_{j}(\cdot)$, and $h_{j}(\cdot)$ denote the activation function between the $j$ th-dimension of the memristor and the state variable; $\tau_{j}(t)$ and $\rho_{j}(t)$, respectively, describe the discrete and distributed time-varying delays which satisfied $0 \leq \tau_{j}(t) \leq \tau_{j}^{\max }$ and
$0 \leq \rho_{j}(t) \leq \rho_{j}^{\max }$; and $I_{i}$ represents the external input or bias, and it is a constant. Furthermore, $a_{i j}\left(x_{i}(t)\right), b_{i j}\left(x_{i}(t)\right)$, and $c_{i j}\left(x_{i}(t)\right)$ are memristive synaptic connection weights which can be given by

$$
\begin{aligned}
& a_{i j}\left(x_{i}(t)\right)=\frac{W_{a i j}}{C_{i}} \times \operatorname{sign}_{i j}, \\
& b_{i j}\left(x_{i}(t)\right)=\frac{W_{b i j}}{C_{i}} \times \operatorname{sign}_{i j}, \\
& c_{i j}\left(x_{i}(t)\right)=\frac{W_{c i j}}{C_{i}} \times \operatorname{sign}_{i j},
\end{aligned}
$$

where $\operatorname{sign}_{i j}=\left\{\begin{array}{ll}1, & i \neq j \\ -1, & i=j\end{array} ; C_{i}\right.$ represents the capacitors; and $W_{a i j}, W_{b i j}$, and $W_{c i j}$, respectively; denote the memductance of memristors by $R_{a i j}, R_{b i j}$, and $R_{c i j}$. And $R_{a i j}$ describes the memristor between activation function $f_{j}\left(x_{j}(t)\right)$ and $x_{i}(t) ; R_{b i j}$ denotes the memristor between activation function $g_{j}\left(x_{j}\left(t-\tau_{j}\right)\right)$ and $x_{i}(t)$; and $R_{c i j}$ is the memristor between activation function $\int_{t-\rho_{j}(t)}^{t} h_{j}\left(x_{j}(s)\right) \mathrm{d} s$ and $x_{i}(t)$. According to the characteristics of memristors and the current-voltage, we use a simplified memristor model as follows:

$$
\begin{aligned}
& a_{i j}\left(x_{i}(t)\right)= \begin{cases}a_{i j}^{*}, & \left|x_{i}(t)\right| \leq \Omega_{i}, \\
a_{i j}^{* *}, & \left|x_{i}(t)\right|>\Omega_{i},\end{cases} \\
& b_{i j}\left(x_{i}(t)\right)= \begin{cases}b_{i j}^{*}, & \left|x_{i}(t)\right| \leq \Omega_{i}, \\
b_{i j}^{* *}, & \left|x_{i}(t)\right|>\Omega_{i},\end{cases} \\
& c_{i j}\left(x_{i}(t)\right)= \begin{cases}c_{i j}^{*}, & \left|x_{i}(t)\right| \leq \Omega_{i}, \\
c_{i j}^{* *}, & \left|x_{i}(t)\right|>\Omega_{i},\end{cases}
\end{aligned}
$$

where the switching jumps $\Omega_{i}>0$ and $a_{i j}^{*}, a_{i j}^{* *}, b_{i j}^{*}, b_{i j}^{* *}, c_{i j}^{*}$, and $c_{i j}^{* *}$ are all constants. And drive system (66) is supplemented with initial conditions as follows:

$$
\begin{aligned}
x_{i}(s) & =\Xi_{i}(s), \\
s & \in\left[t_{0}-\tau, t_{0}\right], \\
\tau & =\max _{1 \leq j \leq n}\left\{\tau_{j}(t), \rho_{j}(t)\right\} .
\end{aligned}
$$

In order to express the proof process clearly, we set some mathematical simplified forms. Let $a_{i j}^{\prime \prime}=\max \left\{a_{i j}^{*}, a_{i j}^{* *}\right\}$, $a_{i j}^{\prime}=\min \left\{a_{i j}^{*}, a_{i j}^{* *}\right\}, \quad b_{i j}^{\prime \prime}=\max \left\{b_{i j}^{*}, b_{i j}^{* *}\right\}, \quad b_{i j}^{\prime}=\min \left\{b_{i j}^{*}, b_{i j}^{* *}\right\}$, $c_{i j}^{\prime \prime}=\max \left\{c_{i j}^{*}, c_{i j}^{* *}\right\}$, and $c_{i j}^{\prime}=\min \left\{c_{i j}^{*}, c_{i j}^{* *}\right\}$. And $\bar{a}_{i j}=(1 / 2)$ $\left(a_{i j}^{\prime \prime}+a_{i j}^{\prime}\right), \quad \tilde{a}_{i j}=(1 / 2)\left(a_{i j}^{\prime \prime}+a_{i j}^{\prime}\right), \quad \bar{b}_{i j}=(1 / 2)\left(b_{i j}^{\prime \prime}+b_{i j}^{\prime}\right)$, 


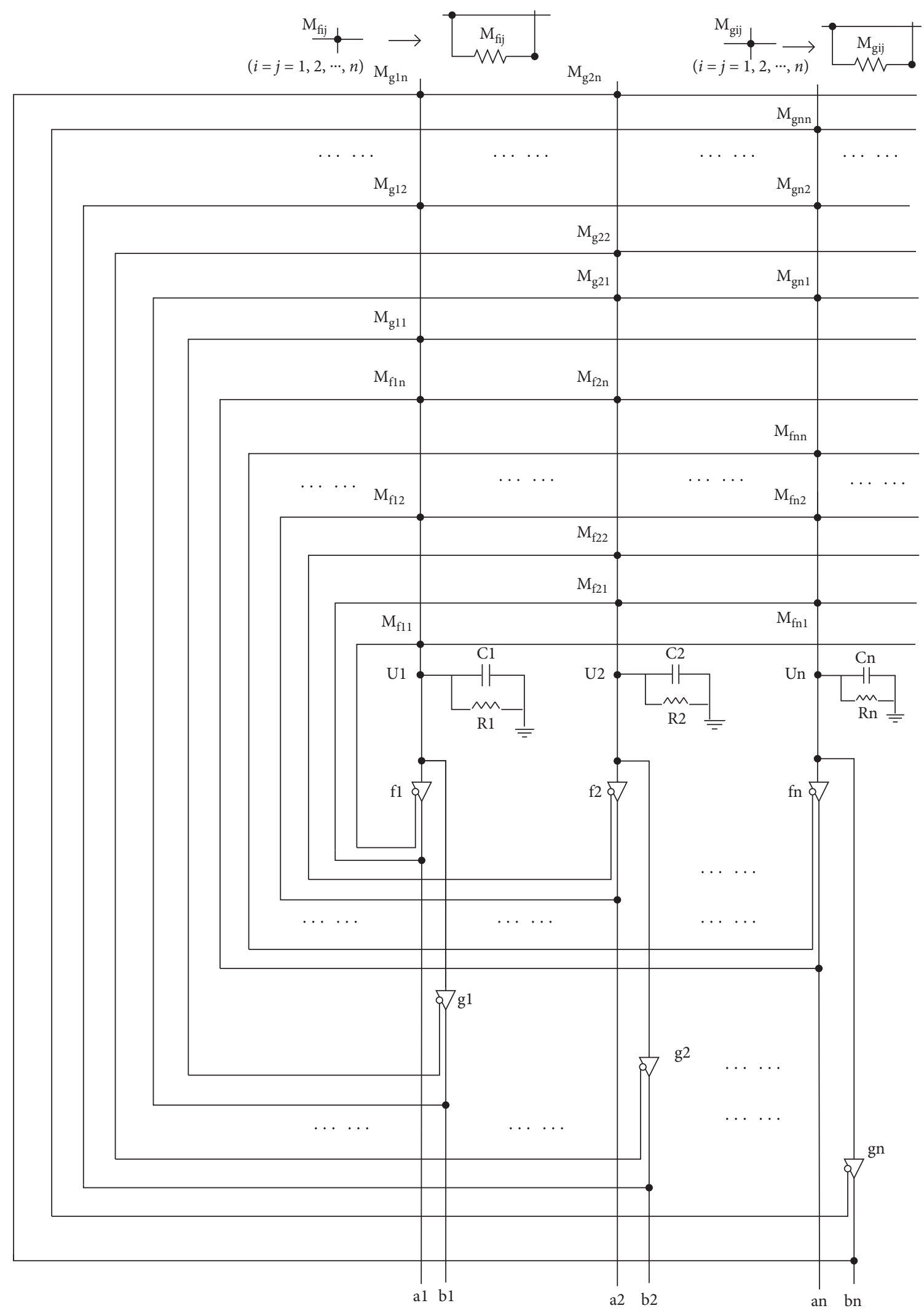

Figure 1: The circuit of the memristive neural network. 
$\widetilde{b}_{i j}=(1 / 2)\left(b_{i j}^{\prime \prime}+b_{i j}^{\prime}\right), \quad \bar{c}_{i j}=(1 / 2)\left(c_{i j}^{\prime \prime}+c_{i j}^{\prime}\right)$, and $\widetilde{c}_{i j}=(1 / 2)$ $\left(c_{i j}^{\prime \prime}+c_{i j}^{\prime}\right)$. Also, $a_{i j}^{+}=\max \left\{\left|a_{i j}^{*}\right|,\left|a_{i j}^{* *}\right|\right\}, b_{i j}^{+}=\max \left\{\left|b_{i j}^{*}\right|,\left|b_{i j}^{* *}\right|\right\}$, and $c_{i j}^{+}=\max \left\{\left|c_{i j}^{*}\right|,\left|c_{i j}^{* *}\right|\right\}$.

From the above description, we note that system (66) can be regarded as a class of the complex switching system with discontinuous right-hand side. In order to discuss the solution of the system, we have to consider them in Filippov's sense. With the theories of the set-valued map and differential inclusions $[48,49]$, drive system (66) could be converted to the following differential inclusion:

$$
\begin{aligned}
& { }_{t_{0}}^{C} D_{t}^{\alpha} x_{i}(t) \epsilon-d_{i} x_{i}(t)+\sum_{j=1}^{n}\left(\bar{a}_{i j}+c o\left[-\widetilde{a}_{i j}, \widetilde{a}_{i j}\right]\right) f_{j}\left(x_{j}(t)\right)+\sum_{j=1}^{n}\left(\bar{b}_{i j}+c o\left[-\widetilde{b}_{i j}, \widetilde{b}_{i j}\right]\right) g_{j}\left(x_{j}\left(t-\tau_{j}(t)\right)\right) \\
& +\sum_{j=1}^{n}\left(\bar{c}_{i j}+c o\left[-\widetilde{c}_{i j}, \widetilde{c}_{i j}\right]\right) \int_{t-\rho_{j}(t)}^{t} h_{j}\left(x_{j}(s)\right) \mathrm{d} s+I_{i}, \quad i=1,2, \ldots, n, t \geq t_{0} .
\end{aligned}
$$

According to the set-valued map and the measurable selection theorem in [50], there exist measurable functions $\delta_{i j}^{(1)}, \delta_{i j}^{(2)}, \delta_{i j}^{(3)} \in c o[-1,1]$ such that

$$
\begin{aligned}
{ }_{t_{0}}^{C} \mathrm{D}_{t}^{\alpha} x_{i}(t)= & -d_{i} x_{i}(t)+\sum_{j=1}^{n}\left(\bar{a}_{i j}+\widetilde{a}_{i j} \delta_{i j}^{(1)}\right) f_{j}\left(x_{j}(t)\right)+\sum_{j=1}^{n}\left(\bar{b}_{i j}+\widetilde{b}_{i j} \delta_{i j}^{(2)}\right) g_{j}\left(x_{j}\left(t-\tau_{j}(t)\right)\right) \\
& +\sum_{j=1}^{n}\left(\bar{c}_{i j}+\widetilde{c}_{i j} \delta_{i j}^{(3)}\right) \int_{t-\rho_{j}(t)}^{t} h_{j}\left(x_{j}(s)\right) \mathrm{d} s+I_{i}, \quad i=1,2, \ldots, n, t \geq t_{0} .
\end{aligned}
$$

In order to investigate the synchronization of FMNNs, system (66) is regarded as the drive system. Similarly, the response system is described as follows:

$$
\begin{aligned}
{ }_{t_{0}}^{C} \mathrm{D}_{t}^{\alpha} y_{i}(t)= & -d_{i} y_{i}(t)+\sum_{j=1}^{n} a_{i j}\left(y_{i}(t)\right) f_{j}\left(y_{j}(t)\right) \\
& +\sum_{j=1}^{n} b_{i j}\left(y_{i}(t)\right) g_{j}\left(y_{j}\left(t-\tau_{j}(t)\right)\right) \\
& +\sum_{j=1}^{n} c_{i j}\left(y_{i}(t)\right) \int_{t-\rho_{j}(t)}^{t} h_{j}\left(y_{j}(s)\right) \mathrm{d} s+I_{i}+u_{i}(t), \quad i=1,2, \ldots, n, t \geq t_{0} .
\end{aligned}
$$

where $y_{i}(t)$ represents the state of the response system and $u_{i}(t)$ is the controller to be designed. And the initial conditions of response system (72) are given as follows:

$$
\begin{aligned}
y_{i}(s) & =\Psi_{i}(s), \\
s & \in\left[t_{0}-\tau, t_{0}\right], \\
\tau & =\max _{1 \leq j \leq n}\left\{\tau_{j}(t), \rho_{j}(t)\right\} .
\end{aligned}
$$

By using the set-valued map and the measurable selection theorem, there exist measurable functions $\gamma_{i j}^{(1)}, \gamma_{i j}^{(2)}, \gamma_{i j}^{(3)} \in \operatorname{co}[-1,1]$ such that 


$$
\begin{aligned}
{ }_{t_{0}}^{C} D_{t}^{\alpha} y_{i}(t)= & -d_{i} y_{i}(t)+\sum_{j=1}^{n}\left(\bar{a}_{i j}+\widetilde{a}_{i j} \gamma_{i j}^{(1)}\right) f_{j}\left(y_{j}(t)\right)+\sum_{j=1}^{n}\left(\bar{b}_{i j}+\widetilde{b}_{i j} \gamma_{i j}^{(2)}\right) g_{j}\left(y_{j}\left(t-\tau_{j}(t)\right)\right) \\
& +\sum_{j=1}^{n}\left(\bar{c}_{i j}+\widetilde{c}_{i j} \gamma_{i j}^{(3)}\right) \int_{t-\rho_{j}(t)}^{t} h_{j}\left(y_{j}(s)\right) \mathrm{d} s+I_{i}+u_{i}(t), \quad i=1,2, \ldots, n, t \geq t_{0} .
\end{aligned}
$$

Remark 3. The drive system and the response system are a distinct network, so $\Delta_{i j}\left(x_{i}(t)\right)$ probably is not equal to $\Delta_{i j}\left(y_{i}(t)\right)$. Thus, we need to select six different measurable functions $\delta_{i j}^{(1)}, \delta_{i j}^{(2)}, \delta_{i j}^{(3)}, \gamma_{i j}^{(1)}, \gamma_{i j}^{(2)}, \gamma_{i j}^{(3)}$.
Let $e_{i}(t)=y_{i}(t)-\lambda x_{i}(t)$ denote the synchronization error. And $\lambda$ represents the projective coefficient. Hence, the fractional order of the synchronization error system can be given as follows:

$$
\begin{aligned}
{ }_{t_{0}}^{C} D_{t}^{\alpha} e_{i}(t)= & -d_{i} e_{i}(t)+(1-\lambda) I_{i}+\sum_{j=1}^{n}\left(\bar{a}_{i j}+\tilde{a}_{i j} \gamma_{i j}^{(1)}\right) f_{j}\left(y_{j}(t)\right)-\lambda \sum_{j=1}^{n}\left(\bar{a}_{i j}+\tilde{a}_{i j} \delta_{i j}^{(1)}\right) f_{j}\left(x_{j}(t)\right) \\
& +\sum_{j=1}^{n}\left(\bar{b}_{i j}+\widetilde{b}_{i j} \gamma_{i j}^{(2)}\right) g_{j}\left(y_{j}\left(t-\tau_{j}(t)\right)\right)-\lambda \sum_{j=1}^{n}\left(\bar{b}_{i j}+\widetilde{b}_{i j} \delta_{i j}^{(2)}\right) g_{j}\left(x_{j}\left(t-\tau_{j}(t)\right)\right) \\
& +\sum_{j=1}^{n}\left(\bar{c}_{i j}+\widetilde{c}_{i j} \gamma_{i j}^{(3)}\right) \int_{t-\rho_{j}(t)}^{t} h_{j}\left(y_{j}(s)\right) \mathrm{d} s-\lambda \sum_{j=1}^{n}\left(\bar{c}_{i j}+\widetilde{c}_{i j} \delta_{i j}^{(3)}\right) \int_{t-\rho_{j}(t)}^{t} h_{j}\left(x_{j}(s)\right) \mathrm{d} s+u_{i}(t) .
\end{aligned}
$$

The initial conditions associated with system (75) are written as follows:

$$
\begin{aligned}
e_{i}(s) & =\Phi_{i}(s)=\Xi_{i}(t)-\lambda \Psi_{i}(s), \\
s & \in\left[t_{0}-\tau, t_{0}\right], \\
\tau & =\max _{1 \leq j \leq n}\left\{\tau_{j}(t), \rho_{j}(t)\right\} .
\end{aligned}
$$

To obtain the main results expediently, we set up the following assumptions:

Assumption 1. The activation functions $f_{j}(\cdot), g_{j}(\cdot)$, and $h_{j}(\cdot)$ are Lipschitz continuous. That is, for any $j \in \mathbb{N}$, there exist three constants $L_{j}^{(1)}, L_{j}^{(2)}$, and $L_{j}^{(3)}$ such that

$$
\mid \begin{aligned}
& f_{j}(y)-f_{j}(x)\left|\leq L_{j}^{(1)}\right| y-x \mid, \\
& g_{j}(y)-f_{j}(x)\left|\leq L_{j}^{(2)}\right| y-x \mid, \\
& h_{j}(y)-h_{j}(x)\left|\leq L_{j}^{(3)}\right| y-x \mid,
\end{aligned}
$$

where $x, y \in \mathbb{R}, x \neq y$.

Assumption 2. The activation functions $f_{j}(\cdot), g_{j}(\cdot)$, and $h_{j}(\cdot)$ are bounded. That is, for any $j \in \mathbb{N}$, there exist three constants $M_{j}^{(1)}, M_{j}^{(2)}$, and $M_{j}^{(3)}$ such that

$$
\begin{aligned}
& \left|f_{j}(x)\right| \leq M_{j}^{(1)}, \\
& \left|g_{j}(x)\right| \leq M_{j}^{(2)}, \\
& \left|h_{j}(x)\right| \leq M_{j}^{(3)},
\end{aligned}
$$

where $x \in \mathbb{R}$.

It is obvious that error system (75) can be expressed as follows: 


$$
\begin{aligned}
{ }_{t_{0}}^{C} D_{t}^{\alpha} e_{i}(t)= & -d_{i} e_{i}(t)+(1-\lambda) I_{i}++u_{i}(t)+\sum_{j=1}^{n}\left(\bar{a}_{i j}+\tilde{a}_{i j} \gamma_{i j}^{(1)}\right)\left[f_{j}\left(y_{j}(t)\right)-f_{j}\left(\lambda x_{j}(t)\right)\right] \\
& +\sum_{j=1}^{n}\left(\bar{a}_{i j}+\tilde{a}_{i j} \gamma_{i j}^{(1)}\right)\left[f_{j}\left(\lambda x_{j}(t)\right)-f_{j}\left(x_{j}(t)\right)\right]+\sum_{j=1}^{n}\left[(1-\lambda) \bar{a}_{i j}+\left(\gamma_{i j}^{(1)}-\lambda \delta_{i j}^{(1)}\right) \tilde{a}_{i j}\right] f_{j}\left(x_{j}(t)\right) \\
& +\sum_{j=1}^{n}\left(\bar{b}_{i j}+\widetilde{b}_{i j} \gamma_{i j}^{(2)}\right)\left[g_{j}\left(y_{j}\left(t-\tau_{j}(t)\right)\right)-g_{j}\left(\lambda x_{j}\left(t-\tau_{j}(t)\right)\right)\right] \\
& +\sum_{j=1}^{n}\left(\bar{b}_{i j}+\widetilde{b}_{i j} \gamma_{i j}^{(2)}\right)\left[g_{j}\left(\lambda x_{j}\left(t-\tau_{j}(t)\right)\right)-g_{j}\left(x_{j}\left(t-\tau_{j}(t)\right)\right)\right] \\
& +\sum_{j=1}^{n}\left[(1-\lambda) \bar{b}_{i j}+\left(\gamma_{i j}^{(2)}-\lambda \delta_{i j}^{(2)}\right) \widetilde{b}_{i j}\right] g_{j}\left(x_{j}\left(t-\tau_{j}(t)\right)\right) \\
& +\sum_{j=1}^{n}\left(\bar{c}_{i j}+\widetilde{c}_{i j} \gamma_{i j}^{(3)}\right) \int_{t-\rho_{j}(t)}^{t} h_{j}\left(y_{j}(s)\right) \mathrm{d} s-\lambda \sum_{j=1}^{n}\left(\bar{c}_{i j}+\widetilde{c}_{i j} \delta_{i j}^{(3)}\right) \int_{t-\rho_{j}(t)}^{t} h_{j}\left(x_{j}(s)\right) \mathrm{d} s \\
& \leq-d_{i} e_{i}(t)+(1-\lambda) I_{i}+u_{i}(t)+\sum_{j=1}^{n} a_{i j}^{+}\left|f_{j}\left(y_{j}(t)\right)-f_{j}\left(\lambda x_{j}(t)\right)\right|+\sum_{j=1}^{n} a_{i j}^{+}\left|f_{j}\left(\lambda x_{j}(t)\right)-f_{j}\left(x_{j}(t)\right)\right| \\
& +\sum_{j=1}^{n}\left|(1-\lambda) \bar{a}_{i j}+\left(\gamma_{i j}^{(1)}-\lambda \delta_{i j}^{(1)}\right) \tilde{a}_{i j}\right| f_{j}\left(x_{j}(t)\right)\left|+\sum_{j=1}^{n} b_{i j}^{+}\right| t g_{j} n\left(y_{j}\left(t-\tau_{j}(t)\right)\right) q-h g_{j}\left(\lambda x_{j}\left(t-\tau_{j}(t)\right)\right) \mid \\
& +\sum_{j=1}^{n} b_{i j}^{+}\left|t g_{j} n\left(\lambda x_{j}\left(t-\tau_{j}(t)\right)\right) q-h g_{j}\left(x_{j}\left(t-\tau_{j}(t)\right)\right)\right|+\sum_{j=1}^{n}\left|(1-\lambda) \bar{b}_{i j}+\left(\gamma_{i j}^{(2)}-\lambda \delta_{i j}^{(2)}\right) \widetilde{b}_{i j}\right|\left|g_{j}\left(x_{j}\left(t-\tau_{j}(t)\right)\right)\right| \\
& +\sum_{j=1}^{n} c_{i j}^{+} \int_{t-\rho_{j}(t)}^{t}\left|h_{j}\left(y_{j}(s)\right)\right| \mathrm{d} s+\sum_{j=1}^{n} c_{i j}^{+}|\lambda| \int_{t-\rho_{j}(t)}^{t}\left|h_{j}\left(x_{j}(s)\right)\right| \mathrm{d} s
\end{aligned}
$$

According to Assumption 1, we can judge

According to Assumption 2, we have

$$
\begin{aligned}
& \sum_{j=1}^{n} a_{i j}^{+}\left|f_{j}\left(y_{j}(t)\right)-f_{j}\left(\lambda x_{j}(t)\right)\right| \leq \sum_{j=1}^{n} a_{i j}^{+} L_{j}^{(1)}\left|e_{j}(t)\right|, \\
& \quad \sum_{j=1}^{n} b_{i j}^{+}\left|g_{j}\left(y_{j}\left(t-\tau_{j}(t)\right)\right)-g_{j}\left(\lambda x_{j}\left(t-\tau_{j}(t)\right)\right)\right| \\
& \quad \leq \sum_{j=1}^{n} b_{i j}^{+} L_{j}^{(2)}\left|e_{j}\left(t-\tau_{j}(t)\right)\right| .
\end{aligned}
$$

$$
\begin{aligned}
& \sum_{j=1}^{n} a_{i j}^{+}\left|f_{j}\left(\lambda x_{j}(t)\right)-f_{j}\left(x_{j}(t)\right)\right| \leq \sum_{j=1}^{n} 2 a_{i j}^{+} M_{j}^{(1)} \\
& \sum_{j=1}^{n}\left|(1-\lambda) \bar{a}_{i j}+\left(\gamma_{i j}^{(1)}-\lambda \delta_{i j}^{(1)}\right) \tilde{a}_{i j}\right|\left|f_{j}\left(x_{j}(t)\right)\right| \leq \sum_{j=1}^{n}\left|(1-\lambda) \bar{a}_{i j}+(1+|\lambda|) \widetilde{a}_{i j}\right| M_{j}^{(1)} \\
& \sum_{j=1}^{n} b_{i j}^{+}\left|g_{j}\left(\lambda x_{j}\left(t-\tau_{j}(t)\right)\right)-g_{j}\left(x_{j}\left(t-\tau_{j}(t)\right)\right)\right| \leq \sum_{j=1}^{n} 2 b_{i j}^{+} M_{j}^{(2)} \\
& \sum_{j=1}^{n}\left|(1-\lambda) \bar{b}_{i j}+\left(\gamma_{i j}^{(2)}-\delta_{i j}^{(2)}\right) \widetilde{b}_{i j}\right|\left|g_{j}\left(x_{j}\left(t-\tau_{j}(t)\right)\right)\right| \leq \sum_{j=1}^{n}\left|(1-\lambda) \bar{b}_{i j}+(1+|\lambda|) \widetilde{b}_{i j}\right| M_{j}^{(2)} \\
& \sum_{j=1}^{n} c_{i j}^{+} \int_{t-\rho_{j}(t)}^{t}\left|h_{j}\left(y_{j}(s)\right)\right| \mathrm{d} s+\sum_{j=1}^{n} c_{i j}^{+}|\lambda| \int_{t-\rho_{j}(t)}^{t}\left|h_{j}\left(x_{j}(s)\right)\right| \mathrm{d} s \leq \sum_{j=1}^{n}(1+|\lambda|) c_{i j}^{+} \rho_{j}^{\max } M_{j}^{(3)} .
\end{aligned}
$$


Substituting inequations (80) and (81) into equation (79), we can obtain

$$
\begin{aligned}
{ }_{t_{0}}^{C} D_{t}^{\alpha} e_{i}(t) \leq & -d_{i} e_{i}(t)+(1-\lambda) I_{i}+u_{i}(t) \\
& +\sum_{j=1}^{n}\left[L_{j}^{(1)} a_{i j}^{+}\left|e_{j}(t)\right|+L_{j}^{(2)} b_{i j}^{+}\left|e_{j}\left(t-\tau_{j}(t)\right)\right|+\left(2 a_{i j}^{+}+\left|(1-\lambda) \bar{a}_{i j}+(1+|\lambda|) \tilde{a}_{i j}\right|\right) M_{j}^{(1)}\right. \\
& \left.+\left(2 b_{i j}^{+}+\left|(1-\lambda) \bar{b}_{i j}+(1+|\lambda|) \widetilde{b}_{i j}\right|\right) M_{j}^{(2)}+(1+|\lambda|) c_{i j}^{+} \rho_{j}^{\max } M_{j}^{(3)}\right], \quad i=1,2, \ldots, n, t \geq t_{0} .
\end{aligned}
$$

\section{Main Results}

In this section, a feedback controller is designed for actualizing finite-time projective synchronization of fractionalorder memristor-based neural networks with mixed timevarying delays. And we choose the following feedback control strategy:

$$
u_{i}(t)=-k_{i} e_{i}(t)-\operatorname{sign}\left(e_{i}(t)\right)\left(h_{i}\left|e_{i}\left(t-\tau_{i}(t)\right)\right|+q_{i}\right)-(1-\lambda) I_{i},
$$

where $k_{i}, h_{i}$, and $q_{i}$ denote the controller parameters.

3.1. The Finite-Time Projective Synchronization of FMNNs with a Lyapunov Function Based on 1-Norm
Theorem 1. When Assumptions 1 and 2 hold, drive system (66) and response system (72) achieve the finite-time projective synchronization under state feedback controller (83) if

$$
\begin{aligned}
& \varphi=\min _{1 \leq i \leq n}\left\{\varphi_{i}\right\}>0, \\
& \delta=\min _{1 \leq i \leq n}\left\{\delta_{i}\right\}>0, \\
& \eta=\sum_{i=1}^{n} \eta_{i}>0,
\end{aligned}
$$

where

$$
\begin{aligned}
\varphi_{i}= & d_{i}+k_{i}-\sum_{j=1}^{n} L_{i}^{(1)} a_{j i}^{+}, \\
\delta_{i}= & h_{i}-\sum_{j=1}^{n} L_{i}^{(2)} b_{j i}^{+}, \\
\eta_{i}= & q_{i}-\sum_{j=1}^{n}\left[\left(2 a_{i j}^{+}+\left|(1-\lambda) \bar{a}_{i j}+(1+|\lambda|) \tilde{a}_{i j}\right|\right) M_{j}^{(1)}\right. \\
& \left.+\left(2 b_{i j}^{+}+\left|(1-\lambda) \bar{b}_{i j}+(1+|\lambda|) \widetilde{b}_{i j}\right|\right) M_{j}^{(2)}+(1+|\lambda|) c_{i j}^{+} \rho_{j}^{\max } M_{j}^{(3)}\right] .
\end{aligned}
$$

Moreover, the settling time $T_{1}$ is estimated by

$$
T_{1}^{*} \leq t_{0}+\varphi^{-1 / \alpha} \ln \frac{\varphi V_{1}\left(t_{0}\right)+\eta}{\alpha \eta} .
$$

Proof. Firstly, we consider the following Lyapunov function:

$$
V_{1}(t)=\sum_{i=1}^{n}\left|e_{i}(t)\right|
$$

Based on Lemma 8, the above fractional-order derivative of $V(t)$ can be written as follows:

$$
{ }_{t_{0}}^{C} D_{t}^{\alpha} V_{1}(t) \leq \sum_{i=1}^{n} \operatorname{sign}\left(e_{i}(t)\right)_{t_{0}}^{C} D_{t}^{\alpha} e_{i}(t) .
$$




$$
\begin{aligned}
{ }_{t_{0}}^{C} D_{t}^{\alpha} V_{1}(t) \leq & \sum_{i=1}^{n} \operatorname{sign}\left(e_{i}(t)\right)_{t_{0}}^{C} D_{t}^{\alpha} e_{i}(t) \\
\leq & \sum_{i=1}^{n} \operatorname{sign}\left(e_{i}(t)\right)\left\{-d_{i} e_{i}(t)+(1-\lambda) I_{i}+u_{i}(t)+\sum_{j=1}^{n}\left[L_{j}^{(1)} a_{i j}^{+}\left|e_{j}(t)\right|+L_{j}^{(2)} b_{i j}^{+}\left|e_{j}\left(t-\tau_{j}(t)\right)\right|\right.\right. \\
& \left.\left.+\left(2 a_{i j}^{+}+\left|(1-\lambda) \bar{a}_{i j}+(1+|\lambda|) \tilde{a}_{i j}\right|\right) M_{j}^{(1)}+\left(2 b_{i j}^{+}+\left|(1-\lambda) \bar{b}_{i j}+(1+|\lambda|) \tilde{b}_{i j}\right|\right) M_{j}^{(2)}+(1+|\lambda|) c_{i j}^{+} \rho_{j}^{\max } M_{j}^{(3)}\right]\right\} \\
\leq & \sum_{i=1}^{n}\left\{-d_{i}\left|e_{i}(t)\right|+\operatorname{sign}\left(e_{i}(t)\right)\left((1-\lambda) I_{i}+u_{i}(t)\right)+\sum_{j=1}^{n} L_{i}^{(1)} a_{j i}^{+}\left|e_{i}(t)\right|+L_{i}^{(2)} b_{j i}^{+}\left|e_{i}\left(t-\tau_{i}(t)\right)\right|\right. \\
& \left.+\left(2 a_{i j}^{+}+\left|(1-\lambda) \bar{a}_{i j}+(1+|\lambda|) \tilde{a}_{i j}\right|\right) M_{j}^{(1)}+\left(2 b_{i j}^{+}+\left|(1-\lambda) \bar{b}_{i j}+(1+|\lambda|) \widetilde{b}_{i j}\right|\right) M_{j}^{(2)}+(1+|\lambda|) c_{i j}^{+} \rho_{j}^{\max } M_{j}^{(3)}\right\} .
\end{aligned}
$$

Substituting the controller (83) into inequation (89),

$$
\begin{aligned}
{ }_{t_{0}}^{C} D_{t}^{\alpha} V_{1}(t) \leq & \sum_{i=1}^{n}\left\{-d_{i}\left|e_{i}(t)\right|+\sum_{j=1}^{n}\left[L_{i}^{(1)} a_{j i}^{+}\left|e_{i}(t)\right|+L_{i}^{(2)} b_{j i}^{+}\left|e_{i}\left(t-\tau_{i}(t)\right)\right|+\left(2 a_{i j}^{+}+\left|(1-\lambda) \bar{a}_{i j}+(1+|\lambda|) \widetilde{a}_{i j}\right|\right) M_{j}^{(1)}\right.\right. \\
& \left.\left.+\left(2 b_{i j}^{+}+\left|(1-\lambda) \bar{b}_{i j}+(1+|\lambda|) \widetilde{b}_{i j}\right|\right) M_{j}^{(2)}+(1+|\lambda|) c_{i j}^{+} \rho_{j}^{\max } M_{j}^{(3)}\right]-k_{i}\left|e_{i}(t)\right|-h_{i}\left|e_{i}\left(t-\tau_{i}(t)\right)\right|-q_{i}\right\} \\
= & -\sum_{i=1}^{n}\left[d_{i}+k_{i}-\sum_{j=1}^{n} L_{i}^{(1)} a_{j i}^{+}\right]\left|e_{i}(t)\right|-\sum_{i=1}^{n}\left(h_{i}-\sum_{j=1}^{n} L_{i}^{(2)} b_{j i}^{+}\right)\left|e_{i}\left(t-\tau_{i}(t)\right)\right| \\
& -\sum_{i=1}^{n}\left\{q_{i}-\sum_{j=1}^{n}\left[\left(2 a_{i j}^{+}+\left|(1-\lambda) \tilde{a}_{i j}+(1+|\lambda|) \widetilde{a}_{i j}\right|\right) M_{j}^{(1)}+\left(2 b_{i j}^{+}+\left|(1-\lambda) \bar{b}_{i j}+(1+|\lambda|) \widetilde{b}_{i j}\right|\right) M_{j}^{(2)}+(1+|\lambda|) c_{i j}^{+} \rho_{j}^{\max } M_{j}^{(3)}\right]\right\} .
\end{aligned}
$$

Let

$$
\begin{aligned}
\varphi_{i}= & d_{i}+k_{i}-\sum_{j=1}^{n} L_{i}^{(1)} a_{j i}^{+}, \\
\delta_{i}= & h_{i}-\sum_{j=1}^{n} L_{i}^{(2)} b_{j i}^{+}, \\
\eta_{i}= & q_{i}-\sum_{j=1}^{n}\left[\left(2 a_{i j}^{+}+\left|(1-\lambda) \bar{a}_{i j}+(1+|\lambda|) \tilde{a}_{i j}\right|\right) M_{j}^{(1)}\right. \\
& \left.+\left(2 b_{i j}^{+}+\left|(1-\lambda) \bar{b}_{i j}+(1+|\lambda|) \tilde{b}_{i j}\right|\right) M_{j}^{(2)}+(1+|\lambda|) c_{i j}^{+} \rho_{j}^{\max } M_{j}^{(3)}\right] .
\end{aligned}
$$

Therefore, in equation (90) can be rewritten as follows:

$$
\begin{array}{r}
{ }_{t_{0}}^{C} D_{t}^{\alpha} V_{1}(t) \leq-\sum_{i=1}^{n} \varphi_{i}\left|e_{i}(t)\right|-\sum_{i=1}^{n} \delta_{i}\left|e_{i}\left(t-\tau_{i}(t)\right)\right|-\sum_{i=1}^{n} \eta_{i} \\
\leq-\varphi \sum_{i=1}^{n}\left|e_{i}(t)\right|-\delta \sum_{i=1}^{n}\left|e_{i}\left(t-\tau_{i}(t)\right)\right|-\eta \\
=-\varphi V_{1}(t)-\delta V_{1}\left(t-\tau_{i}(t)\right)-\eta
\end{array}
$$

where $\varphi=\min _{1 \leq i \leq n}\left\{\varphi_{i}\right\}, \delta=\min _{1 \leq i \leq n}\left\{\delta_{i}\right\}$, and $\eta=\sum_{i=1}^{n} \eta_{i}$.
Based on the condition $\delta>0$ in Theorem 1, we have

$$
{ }_{t_{0}}^{C} D_{t}^{\alpha} V_{1}(t) \leq-\varphi V_{1}(t)-\eta,
$$

According to the conditions of Theorem 1, we can gain $\varphi>0, \eta>0$. Furthermore, by virtue of Property 1, it follows that

$$
V_{1}(t) \leq\left[V_{1}\left(t_{0}\right)+\frac{\eta}{\varphi}\right] \frac{1}{\alpha} \exp \left\{-\varphi^{1 / \alpha}\left(t-t_{0}\right)\right\}-\frac{\eta}{\varphi}, \quad t \geq t_{0} .
$$

And we can judge that the Lyapunov function $V_{1}(t)$ converges to 0 , which represents drive system (66) and response system (72) actualizing finite-time projective synchronization under feedback controller (83). And the settling time $T_{1}$ which can be estimated is as follows:

$$
T_{1}^{*} \leq t_{0}+\varphi^{-1 / \alpha} \ln \frac{\varphi V_{1}\left(t_{0}\right)+\eta}{\eta \alpha} .
$$

This completes the proof.

Remark 4. For the differential inequation ${ }_{t_{0}}^{C} D_{t}^{\alpha} V_{1}(t) \leq-$ $\varphi V_{1}(t)-\eta$ in fractional-order neural networks, Zhang et al. 
[29] ignored the effort of parameter $\varphi$ when they calculated the settling time.

$$
{ }_{t_{0}}^{C} D_{t}^{\alpha} V_{1}(t) \leq-\eta \text {. }
$$

Taking the fractional integral with variable order $\alpha$ on both sides of the above inequation, we can gain

$$
V_{1}(t)-V_{1}\left(t_{0}\right) \leq \frac{-1}{\Gamma(\alpha)} \int_{t_{0}}^{t}(t-s)^{\alpha-1} \eta \mathrm{d} s .
$$

Through Definition 1, we can calculate that $V(t)$ converges to 0 within the settling time $T_{2}$, and $T_{2}$ is evaluated by

$$
T_{2} \leq t_{0}+\left[\frac{V_{1}\left(t_{0}\right) \Gamma(1+\alpha)}{\eta}\right]^{1 / \alpha} .
$$

Apparently, the authors ignored the impact of parameter $\eta$, and let $\eta=0$ which will increase the conservation of the settling time. Considering the parameter $\eta>0$; the estimation of settling time $T_{1}^{*}$ in Theorem 1 is less conservative than the results in [29]. To verify that the settling time $T_{1}^{*}$ is more accurate than $T_{2}$, a comparison table has been given in numerical simulation.

Remark 5. For the differential inequation ${ }_{t_{0}}^{C} D_{t}^{\alpha} V_{1}(t) \leq-\varphi V_{1}(t)-\eta$, when fractional order $\alpha=1$, the settling time $t_{1}$ is estimated by

$$
t_{1} \leq t_{0}+\frac{\ln \left[\varphi V_{1}\left(t_{0}\right)\right]-\ln \eta}{\varphi} .
$$

Obviously, the estimate time $t_{1}$ is consistent with the result of the integer-order system given in [51]. Thus, it shows the estimation of settling time $T_{1}^{*}$ in Theorem 1 is more accurate and general.

3.2. The Finite-Time Projective Synchronization of FMNNs with a Lyapunov Function Based on p-Norm

Theorem 2. Under the state feedback controller (83) and Assumptions 1 and 2, drive system (66) and response system (72) achieve the finite-time projective synchronization if

$$
\begin{aligned}
\bar{\varphi}= & \min _{1 \leq i \leq n}\left\{\bar{\varphi}_{i}\right\}>0, \\
\bar{\delta}= & \min _{1 \leq i \leq n}\left\{\bar{\delta}_{i}\right\}>0, \\
\bar{\eta}= & \min _{1 \leq i \leq n}\left\{\bar{\eta}_{i}\right\}>0, \\
\bar{\varphi}_{i}= & \frac{\Gamma(2-\alpha) \Gamma(1+p)}{\Gamma(1+p-\alpha)}\left[d_{i}+k_{i}-\sum_{j=1}^{n}\left(L_{j}^{(1)} a_{i j}^{+} \frac{(p-1) \varepsilon_{1}}{p}+\frac{L_{i}^{(1)} a_{j i}^{+}}{p \varepsilon_{1}^{p-1}}+L_{j}^{(2)} b_{i j}^{+} \frac{(p-1) \varepsilon_{2}}{p}\right)\right], \\
\bar{\delta}_{i}= & \frac{\Gamma(2-\alpha) \Gamma(1+p)}{\Gamma(1+p-\alpha)}\left(h_{i}-\sum_{j=1}^{n} \frac{L_{i}^{(2)} b_{j i}^{+}}{p \varepsilon_{2}^{p-1}}\right), \\
\bar{\eta}_{i}= & \frac{\Gamma(2-\alpha) \Gamma(1+p)}{\Gamma(1+p-\alpha)}\left\{q_{i}-\sum_{j=1}^{n}\left[\left(2 a_{i j}^{+}+\left|(1-\lambda) \bar{a}_{i j}+(1+|\lambda|) \widetilde{a}_{i j}\right|\right) M_{j}^{(1)}\right.\right. \\
& \left.\left.+\left(2 b_{i j}^{+}+\left|(1-\lambda) \bar{b}_{i j}+(1+|\lambda|) \widetilde{b}_{i j}\right|\right) M_{j}^{(2)}+(1+|\lambda|) c_{i j}^{+} \rho_{j}^{\max } M_{j}^{(3)}\right]\right\},
\end{aligned}
$$

where $\varepsilon_{1}$ and $\varepsilon_{2}$ are arbitrary positive constants.

Moreover, the settling time $T_{2}$ is estimated by

$$
T_{3}^{*} \leq t_{0}+\left[\frac{\Gamma(1+(p /(p-1))-\alpha) \bar{\varphi}}{\Gamma(1+(p /(p-1))) \Gamma(2-\alpha)}\right]^{-1 / \alpha} \ln \frac{\bar{\varphi} V_{2}^{1 / p}\left(t_{0}\right)+\bar{\eta}}{\bar{\eta} \alpha} .
$$

Proof. Firstly, we consider the following Lyapunov function:

$$
V_{2}(t)=\sum_{i=1}^{n}\left|e_{i}(t)\right|^{p}
$$

where $p$ is a positive integer with $p>1$.

Calculating the derivate of $V_{2}(t)$ and using Lemma 7 and error system (82), one has

$$
{ }_{t_{0}}^{C} D_{t}^{\alpha} V_{2}(t)=\frac{\Gamma(2-\alpha) \Gamma(1+p)}{\Gamma(1+p-\alpha)} \sum_{i=1}^{n}\left|e_{i}(t)\right|^{p-1}{ }_{t_{0}} D_{t}^{\alpha}\left|e_{i}(t)\right| .
$$

According to Lemma 8, the above fractional-order derivative of $V(t)$ can be written as follows: 


$$
\begin{aligned}
{ }_{t_{0}}^{C} D_{t}^{\alpha} V_{2}(t) \leq & \frac{\Gamma(2-\alpha) \Gamma(1+p)}{\Gamma(1+p-\alpha)} \sum_{i=1}^{n}\left|e_{i}(t)\right|^{p-1} \operatorname{sign}\left(e_{i}(t)\right)_{t_{0}}^{C} D_{t}^{\alpha} e_{i}(t) \\
\leq & \frac{\Gamma(2-\alpha) \Gamma(1+p)}{\Gamma(1+p-\alpha)} \sum_{i=1}^{n}\left\{-d_{i}\left|e_{i}(t)\right|^{p}+\operatorname{sign}\left(e_{i}(t)\right)\left((1-\lambda) I_{i}+u_{i}(t)\right)\left|e_{i}(t)\right|^{p-1}\right. \\
& +\sum_{j=1}^{n}\left[L_{i}^{(1)} a_{i j}^{+}\left|e_{j}(t)\right|+L_{i}^{(2)} b_{i j}^{+}\left|e_{j}\left(t-\tau_{j}(t)\right)\right|+\left(2 a_{i j}^{+}+\left|(1-\lambda) \bar{a}_{i j}+(1+|\lambda|) \tilde{a}_{i j}\right|\right) M_{j}^{(1)}\right. \\
& \left.\left.+\left(2 b_{i j}^{+}+\left|(1-\lambda) \bar{b}_{i j}+(1+|\lambda|) \widetilde{b}_{i j}\right|\right) M_{j}^{(2)}+(1+|\lambda|) c_{i j}^{+} \rho_{j}^{\max } M_{j}^{(3)}\right]\left|e_{i}(t)\right|^{p-1}\right\} .
\end{aligned}
$$

Substituting the feedback controller (83) into inequation (104), we can get

$$
\begin{aligned}
{ }_{t_{0}}^{C} D_{t}^{\alpha} V_{2}(t) \leq & \frac{\Gamma(2-\alpha) \Gamma(1+p)}{\Gamma(1+p-\alpha)} \sum_{i=1}^{n}\left\{-d_{i}\left|e_{i}(t)\right|^{p}+\sum_{j=1}^{n}\left[L_{j}^{(1)} a_{i j}^{+}\left|e_{i}(t)\right|^{p-1}\left|e_{j}(t)\right|+L_{j}^{(2)} b_{i j}^{+}\left|e_{i}(t)\right|^{p-1}\left|e_{j}\left(t-\tau_{j}(t)\right)\right|\right]\right. \\
& +\sum_{j=1}^{n}\left[\left(2 a_{i j}^{+}+\left|(1-\lambda) \bar{a}_{i j}+(1+|\lambda|) \widetilde{a}_{i j}\right|\right) M_{j}^{(1)}+\left(2 b_{i j}^{+}+\left|(1-\lambda) \bar{b}_{i j}+(1+|\lambda|) \widetilde{b}_{i j}\right|\right) M_{j}^{(2)}\right. \\
& \left.\left.+(1+|\lambda|) c_{i j}^{+} \rho_{j}^{\max } M_{j}^{(3)}\right]\left|e_{i}(t)\right|^{p-1}-k_{i}\left|e_{i}(t)\right|^{p}-h_{i}\left|e_{i}\left(t-\tau_{i}(t)\right)\right|^{p}-q_{i}\left|e_{i}(t)\right|^{p-1}\right\} .
\end{aligned}
$$

By applying Lemma 3, we have

$$
\left|e_{i}(t)\right|^{p-1}\left|e_{j}(t)\right| \leq \frac{(p-1) \varepsilon_{1}}{p}\left|e_{i}(t)\right|^{p}+\frac{1}{p \varepsilon_{1}^{p-1}}\left|e_{j}(t)\right|^{p},
$$

$\left|e_{i}(t)\right|^{p-1}\left|e_{j}\left(t-\tau_{j}(t)\right)\right| \leq \frac{(p-1) \varepsilon_{2}}{p}\left|e_{i}(t)\right|^{p}+\frac{1}{p \varepsilon_{2}^{p-1}}\left|e_{j}\left(t-\tau_{j}(t)\right)\right|^{p}$, where $\varepsilon_{1}$ and $\varepsilon_{2}$ are arbitrary positive constants.

Combining with inequations (105) and (106), one has 


$$
\begin{aligned}
{ }_{t_{0}}^{C} D t \alpha V_{1}(t) \leq & \frac{\Gamma(2-\alpha) \Gamma(1+p)}{\Gamma(1+p-\alpha)} \sum_{i=1}^{n}\left\{-\left(d_{i}+k_{i}\right)\left|e_{i}(t)\right|^{p}-h_{i}\left|e_{i}\left(t-\tau_{i}(t)\right)\right|^{p}-q_{i}\left|e_{i}(t)\right|^{p-1}\right. \\
& +\sum_{j=1}^{n}\left(L_{j}^{(1)} a_{i j}^{+} \frac{(p-1) \varepsilon_{1}}{p}+\frac{L_{i}^{(1)} a_{j i}^{+}}{p \varepsilon_{1}^{p-1}}+L_{j}^{(2)} b_{i j}^{+} \frac{(p-1) \varepsilon_{2}}{p}\right)\left|e_{i}(t)\right|^{p}+\sum_{j=1}^{n} \frac{L_{i}^{(2)} b_{j i}^{+}}{p \varepsilon_{2}^{p-1}\left|e_{i}\left(t-\tau_{i}(t)\right)\right|^{p}} \\
& \left.+\sum_{j=1}^{n}\left[\left(2 a_{i j}^{+}+\left|(1-\lambda) \bar{a}_{i j}+(1+|\lambda|) \widetilde{a}_{i j}\right|\right) M_{j}^{(1)}+\left(2 b_{i j}^{+}+\left|(1-\lambda) \bar{b}_{i j}+(1+|\lambda|) \widetilde{b}_{i j}\right|\right) M_{j}^{(2)}+(1+|\lambda|) c_{i j}^{+} \rho_{j}^{\max } M_{j}^{(3)}\right]\left|e_{i}(t)\right|^{p-1}\right\} \\
= & -\frac{\Gamma(2-\alpha) \Gamma(1+p)}{\Gamma(1+p-\alpha)} \sum_{i=1}^{n}\left[d_{i}+k_{i}-\sum_{j=1}^{n}\left(L_{j}^{(1)} a_{i j}^{+} \frac{(p-1) \varepsilon_{1}}{p}+\frac{L_{i}^{(1)} a_{j i}^{+}}{p \varepsilon_{1}^{p-1}}+L_{j}^{(2)} b_{i j}^{+} \frac{(p-1) \varepsilon_{2}}{p}\right)\right]\left|e_{i}(t)\right|^{p} \\
& -\frac{\Gamma(2-\alpha) \Gamma(1+p)}{\Gamma(1+p-\alpha)} \sum_{i=1}^{n}\left(h_{i}-\sum_{j=1}^{n} \frac{L_{i}^{(2)} b_{j i}^{+}}{p \varepsilon_{2}^{p-1}}\right)\left|e_{i}\left(t-\tau_{i}(t)\right)\right|^{p} \\
& -\frac{\Gamma(2-\alpha) \Gamma(1+p)}{\Gamma(1+p-\alpha)} \sum_{i=1}^{n}\left\{q_{i}-\sum_{j=1}^{n}\left[\left(2 a_{i j}^{+}+\left|(1-\lambda) \bar{a}_{i j}+(1+|\lambda|) \tilde{a}_{i j}\right|\right) M_{j}^{(1)}\right.\right. \\
& \left.\left.+\left(2 b_{i j}^{+}+\left|(1-\lambda) \bar{b}_{i j}+(1+|\lambda|) \widetilde{b}_{i j}\right|\right) M_{j}^{(2)}+(1+|\lambda|) c_{i j}^{+} \rho_{j}^{\max } M_{j}^{(3)}\right]\right\}\left|e_{i}(t)\right|^{p-1} \\
\leq & -\sum_{i=1}^{n} \bar{\varphi}_{i}\left|e_{i}(t)\right|^{p}-\sum_{i=1}^{n} \bar{\delta}_{i}\left|e_{i}\left(t-\tau_{j}(t)\right)\right|^{p}-\sum_{i=1}^{n} \bar{\eta}_{i}\left|e_{i}(t)\right|^{p-1},
\end{aligned}
$$

where

$$
\begin{aligned}
\bar{\varphi}_{i}= & \frac{\Gamma(2-\alpha) \Gamma(1+p)}{\Gamma(1+p-\alpha)}\left[d_{i}+k_{i}-\sum_{j=1}^{n}\left(L_{j}^{(1)} a_{i j}^{+} \frac{(p-1) \varepsilon_{1}}{p}+\frac{L_{i}^{(1)} a_{j i}^{+}}{p \varepsilon_{1}^{p-1}}+L_{j}^{(2)} b_{i j}^{+} \frac{(p-1) \varepsilon_{2}}{p}\right)\right], \\
\bar{\delta}_{i}= & \frac{\Gamma(2-\alpha) \Gamma(1+p)}{\Gamma(1+p-\alpha)}\left(h_{i}-\sum_{j=1}^{n} \frac{L_{i}^{(2)} b_{j i}^{+}}{p \varepsilon_{2}^{p-1}}\right), \\
\bar{\eta}_{i}= & \frac{\Gamma(2-\alpha) \Gamma(1+p)}{\Gamma(1+p-\alpha)}\left\{q_{i}-\sum_{j=1}^{n}\left[\left(2 a_{i j}^{+}+\left|(1-\lambda) \bar{a}_{i j}+(1+|\lambda|) \widetilde{a}_{i j}\right|\right) M_{j}^{(1)}\right.\right. \\
& \left.\left.+\left(2 b_{i j}^{+}+\left|(1-\lambda) \bar{b}_{i j}+(1+|\lambda|) \widetilde{b}_{i j}\right|\right) M_{j}^{(2)}+(1+|\lambda|) c_{i j}^{+} \rho_{j}^{\max } M_{j}^{(3)}\right]\right\} .
\end{aligned}
$$
Denote $\bar{\varphi}=\min _{1 \leq i \leq n}\left\{\bar{\varphi}_{i}\right\}, \quad \bar{\delta}=\min _{1 \leq i \leq n}\left\{\bar{\delta}_{i}\right\}, \quad$ and $\quad{ }_{t_{0}}^{C} D_{t}^{\alpha} V_{2}(t) \leq-\bar{\varphi} \sum_{i=1}^{n}\left|e_{i}(t)\right|^{p}-\bar{\delta} \sum_{i=1}^{n}\left|e_{i}\left(t-\tau_{i}(t)\right)\right|^{p}-\bar{\eta}\left(\sum_{i=1}^{n}\left|e_{i}(t)\right|^{p}\right)^{(p-1) / p}$
$\bar{\eta}=\min _{1 \leq i \leq n}\left\{\bar{\eta}_{i}\right\} ;$ one gets

${ }_{t_{0}}^{C} D_{t}^{\alpha} V_{2}(t) \leq-\bar{\varphi} \sum_{i=1}^{n}\left|e_{i}(t)\right|^{p}-\bar{\delta} \sum_{i=1}^{n}\left|e_{i}\left(t-\tau_{i}(t)\right)\right|^{p}-\bar{\eta} \sum_{i=1}^{n}\left|e_{i}(t)\right|^{p-1}$.

$$
=-\bar{\varphi} V_{2}(t)-\bar{\delta} V_{2}\left(t-\tau_{i}(t)\right)-\bar{\eta} V_{2}(t)^{(p-1) / p} .
$$

Applying Lemma 4, we have

Based on the conditions of Theorem 2, we know $\bar{\varphi}>0$, $\bar{\delta}>0$, and $\bar{\eta}>0$. Thus,

$$
{ }_{t_{0}}^{C} D_{t}^{\alpha} V_{2}(t) \leq-\bar{\varphi} V_{2}(t)-\bar{\eta} V_{2}^{(p-1) / p}(t) .
$$


Hence, from Property 3, we have

$$
V_{2}^{1 / p}(t) \leq\left[V_{2}^{1 / p}\left(t_{0}\right)+\frac{\bar{\eta}}{\bar{\varphi}}\right] \frac{1}{\alpha} \exp \left\{-\left(\frac{a \Gamma(1+(p /(p-1))-\alpha)}{\Gamma(1+(p /(p-1))) \Gamma(2-\alpha)}\right)^{1 / \alpha}\left(t-t_{0}\right)\right\}-\frac{\bar{\eta}}{\bar{\varphi}}
$$

And we can judge that the Lyapunov function $V_{2}(t)$ converges to 0 , which represents drive system (66) and response system (72) achieve finite-time projective synchronization under feedback controller (83). And the settling time $T_{3}^{*}$ can be estimated by

$$
T_{3}^{*} \leq t_{0}+\left[\frac{\Gamma(1+(p /(p-1))-\alpha) \bar{\varphi}}{\Gamma(1+(p /(p-1))) \Gamma(2-\alpha)}\right]^{-(1 / \alpha)} \ln \frac{\bar{\varphi} V_{2}^{1 / p}\left(t_{0}\right)+\bar{\eta}}{\bar{\eta} \alpha} .
$$

The proof is accomplished.

Remark 6. For the differential inequation ${ }_{t_{0}}^{C} D_{t}^{\alpha} V_{2}(t) \leq$ $-\bar{\varphi} V_{2}(t)-\bar{\eta} V_{2}^{(p-1) / p}(t)$, considering the parameter $\bar{\varphi}=0$, then

$$
{ }_{t_{0}}^{C} D_{t}^{\alpha} V_{2}(t) \leq-\bar{\eta} V_{2}^{(p-1) / p}(t)
$$

where $\bar{\eta}>0$.

According to Corollary 1, it has

$$
V_{2}(t) \leq\left[V_{2}^{1 / p}\left(t_{0}\right)-\bar{\eta} \cdot \frac{a \Gamma(1+p-\alpha)\left(t-t_{0}\right)^{\alpha}}{\Gamma(1+p) \Gamma(2-\alpha) \Gamma(1+\alpha)}\right]^{1 /(1-\beta)} .
$$

And we can judge that the Lyapunov function $V_{2}(t)$ converges to 0 . Furthermore, the settling time

$$
T_{4} \leq t_{0}+\left[V_{2}^{(1 / p)}\left(t_{0}\right) \frac{\Gamma(1+p) \Gamma(2-\alpha) \Gamma(1+\alpha)}{\Gamma(1+p-\alpha) \bar{\eta}}\right]^{(1 / \alpha)} .
$$

From Remark 2, if $((p-1) / p)<\alpha(1+\alpha)-1$ holds, we can obtain another estimation of settling time:

$$
T_{5} \leq t_{0}+\left[V_{2}^{\alpha(1+\alpha)+(1 / p)-2}\left(t_{0}\right) \frac{\Gamma(1+\alpha) \Gamma(1-((2 p-1) / p(1+\alpha)))}{\bar{\eta} \cdot \Gamma(2+\alpha) \Gamma(1+\alpha-((2 p-1) / p(1+\alpha)))}\right]^{(1 / \alpha)} .
$$

Obviously, compared with $T_{3}^{*}$ in Theorem 2, the estimate times $T_{4}$ and $T_{5}$ are conservative estimations of settling time. And a comparison table about $T_{3}^{*}, T_{4}$, and $T_{5}$ has been given in numerical simulation.

\section{Numerical Simulation}

In this section, several numerical examples are given to illustrate the effectiveness of the obtained results. We consider the following two-dimensional fractional-order memristive neural networks with mixed time-varying delays:

$$
\begin{aligned}
& { }_{t_{0}}^{C} \mathrm{D}_{t}^{0.98} x_{i}(t)=-d_{i} x_{i}(t)+\sum_{j=1}^{2} a_{i j}\left(x_{i}(t)\right) f_{j}\left(x_{j}(t)\right)+\sum_{j=1}^{2} b_{i j}\left(x_{i}(t)\right) g_{j}\left(x_{j}\left(t-\tau_{j}(t)\right)\right)+\sum_{j=1}^{2} c_{i j}\left(x_{i}(t)\right) \int_{t-\rho_{j}(t)}^{t} h_{j}\left(x_{j}(s)\right) \mathrm{d} s+I_{i}, \\
& { }_{t_{0}}^{C} \mathrm{D}_{t}^{0.98} y_{i}(t)=-d_{i} y_{i}(t)+\sum_{j=1}^{2} a_{i j}\left(y_{i}(t)\right) f_{j}\left(y_{j}(t)\right)+\sum_{j=1}^{2} b_{i j}\left(y_{i}(t)\right) g_{j}\left(y_{j}\left(t-\tau_{j}(t)\right)\right)+\sum_{j=1}^{2} c_{i j}\left(y_{i}(t)\right) \int_{t-\rho_{j}(t)}^{t} h_{j}\left(y_{j}(s)\right) \mathrm{d} s+I_{i}+u_{i}(t),
\end{aligned}
$$

where $i=1,2$. And the values of the memristors are given as follows: 


$$
\begin{aligned}
& a_{11}\left(x_{1}(t)\right)= \begin{cases}0.3, & \left|x_{1}(t)\right| \leq 1, \\
0.2, & \left|x_{1}(t)\right|>1,\end{cases} \\
& a_{12}\left(x_{1}(t)\right)= \begin{cases}-0.4, & \left|x_{1}(t)\right| \leq 1, \\
-0.22, & \left|x_{1}(t)\right|>1,\end{cases} \\
& a_{21}\left(x_{2}(t)\right)= \begin{cases}-0.9, & \left|x_{2}(t)\right| \leq 1, \\
-0.8, & \left|x_{2}(t)\right|>1,\end{cases} \\
& a_{22}\left(x_{2}(t)\right)= \begin{cases}0.5, & \left|x_{2}(t)\right| \leq 1, \\
0.8, & \left|x_{2}(t)\right|>1,\end{cases} \\
& b_{11}\left(x_{1}(t)\right)= \begin{cases}-0.6, & \left|x_{1}(t)\right| \leq 1, \\
-0.2, & \left|x_{1}(t)\right|>1,\end{cases} \\
& b_{12}\left(x_{1}(t)\right)= \begin{cases}0.5, & \left|x_{1}(t)\right| \leq 1, \\
0.5, & \left|x_{1}(t)\right|>1,\end{cases} \\
& c_{22}\left(x_{2}(t)\right)= \begin{cases}-0.85, & \left|x_{2}(t)\right| \leq 1, \\
-0.95, & \left|x_{2}(t)\right|>1 .\end{cases} \\
& c_{12}\left(x_{1}(t)\right)= \begin{cases}0.7, & \left|x_{1}(t)\right| \leq 1, \\
0.8, & \left|x_{1}(t)\right|>1,\end{cases}
\end{aligned}
$$

For drive-response system (118), we select the system parameters as follows: $I_{1}=I_{2}=0.1, d_{1}=d_{2}=0.1, \tau_{1}(t)=$ $e^{t} /\left(e^{t}+1\right), \quad \tau_{2}(t)=0.8 * e^{t} /\left(e^{t}+1\right), \quad \rho_{1}(t)=0.5 \cos (t)+$ 0.5 , and $\rho_{2}(t)=0.4 \sin (t)+0.4$. Hence, we can obtain that $\tau_{1}^{\max }=1, \tau_{2}^{\max }=0.8, \rho_{1}^{\max }=1, \rho_{2}^{\max }$

$=0.8$, and $\tau=\max _{j=1,2}\left\{\tau_{j}(t), \rho_{j}(t)\right\}=1$.And the initial values $x(s)=[-1.5,2.5]^{T}, y(s)=[2.5,-0.5]^{T}, \forall s \in[-1,0]$.

The activations are adopted as follows:

$$
f_{j}\left(x_{j}(t)\right)=g_{j}\left(x_{j}(t)\right)=h_{j}\left(x_{j}(t)\right)=\frac{1-e^{-x_{j}(t)}}{1+e^{-x_{j}(t)}}, \quad j=1,2 \text {. }
$$

It is easy to check that Assumption 1 and 2 are satisfied. By simple calculation, $L_{j}^{(1)}=L_{j}^{(2)}=L_{j}^{(3)}=M_{j}^{(1)}=$ $M_{j}^{(2)}=M_{j}^{(3)}=1$, for $j=1,2$.
4.1. Projective Synchronization. First, we choose the projective coefficient $\lambda=2$. We can get the initial values $\Phi_{1}(s)=y_{1}(s)-2 x_{1}(s)=5.5, \quad \Phi_{2}(s)=y_{2}(s)-2 x_{2}(s)=$ $-5.5, V_{1}\left(t_{0}\right)=11$, and $V_{2}\left(t_{0}\right)=60.5$.

According to Theorem 1, it can be calculated as follows:

$$
\begin{aligned}
& \varphi_{1}=k_{1}-1.1, \varphi_{2}=k_{2}-1.1, \\
& \delta_{1}=h_{1}-1, \delta_{2}=h_{2}-0.9, \\
& \eta_{1}=q_{1}-8.9, \eta_{2}=q_{2}-10.38 .
\end{aligned}
$$

And we choose $k_{1}=3.1, k_{2}=3.1, h_{1}=1, h_{2}=0.9, q_{1}=$ 9.9, and $q_{2}=11.38$ which make $\varphi=2$ and $\eta=2$.

Then, we have a finite-time fractional-order differential inequation as follows:

$$
{ }_{t_{0}}^{C} D_{t}^{\alpha} V_{1}(t) \leq-2 V_{1}(t)-2 .
$$

Based on the result in Theorem 1, drive-response system (118) could achieve synchronization in finite time. And we can calculate the setting time $T_{1}^{*}$ as follows:

$$
T_{1}^{*} \leq t_{0}+\varphi^{-1 / \alpha} \ln \frac{\varphi V_{1}\left(t_{0}\right)+\eta}{\alpha \eta} \leq 2^{-1 / 0.98} \ln \left(\frac{2 \times 11+2}{0.98 \times 2}\right)=1.235 .
$$

To verify Remark 4, we can calculate $T_{2}$ to demonstrate that $T_{1}^{*}$ is less conservative than $T_{2}$. It follows that

$$
T_{2} \leq t_{0}+\left[\frac{V_{1}\left(t_{0}\right) \Gamma(1+\alpha)}{\eta}\right]^{1 / \alpha}=\left(\frac{11 \cdot \Gamma(1.98)}{2}\right)^{1 / \alpha}=5.5778
$$

On the contrary, we select $p=2$ and $\varepsilon_{1}=\varepsilon_{2}=1$ for Theorem 2. And it is easy to calculate

$$
\begin{array}{ll}
\bar{\varphi}_{1}=1.9608\left(k_{1}-1.4\right), & \bar{\varphi}_{2}=1.9608\left(k_{2}-1.85\right) \\
\bar{\delta}_{1}=1.9608\left(h_{1}-0.5\right), & \bar{\delta}_{2}=1.9608\left(h_{2}-0.45\right) \\
\bar{\eta}_{1}=1.9608\left(q_{1}-8.9\right), & \bar{\eta}_{2}=1.9608\left(q_{2}-10.38\right)
\end{array}
$$

Under the controller parameter, $k_{1}=3.1, k_{2}=3.1, h_{1}=$ $1, h_{2}=0.9, q_{1}=9.9$, and $q_{2}=11.38$ which lead to $\bar{\varphi}=2.451$ and $\bar{\eta}=1.9608$.

Hence, we have another finite-time fractional-order differential inequation as follows:

$$
{ }_{t_{0}}^{C} D_{t}^{\alpha} V_{2}(t) \leq-2.451 V_{2}(t)-1.9608 V_{2}^{1 / 2}(t) \text {. }
$$

Based on the result in Theorem 2, we can estimate the setting time $T_{3}$ as follows:

$$
T_{3}^{*} \leq t_{0}+\left[\frac{\Gamma(1+(p /(p-1))-\alpha) \bar{\varphi}}{\Gamma(1+(p /(p-1))) \Gamma(2-\alpha)}\right]^{-1 / \alpha} \ln \frac{\bar{\varphi} V_{2}^{1 / p}\left(t_{0}\right)+\bar{\eta}}{\bar{\eta} \alpha}=\left[\frac{2.451 \Gamma(2.02)}{\Gamma(3) \Gamma(1.02)}\right]^{-1 / 0.98} \ln \frac{2.451 \times \sqrt{60.5}+1.9608}{1.9608 \times 0.98}=1.9054
$$


To verify Remark 6, we can calculate $T_{4}$ to demonstrate that $T_{3}^{*}$ is less conservative than $T_{4}$. It follows that

$$
\begin{aligned}
T_{4} & \leq t_{0}+\left[V_{2}^{\frac{1}{p}}\left(t_{0}\right) \frac{\Gamma(1+p) \Gamma(2-\alpha) \Gamma(1+\alpha)}{\Gamma(1+p-\alpha) \bar{\eta}}\right]^{1 / \alpha} \\
& =\left[\sqrt{60.5} \cdot \frac{\Gamma(3) \Gamma(1.02) \Gamma(1.98)}{1.9608 \Gamma(2.02)}\right]^{1 / 0.98}=8.0421 .
\end{aligned}
$$

Considering $p=2$ and $\alpha=0.98,((p-1) / p)<\alpha(1+\alpha)-$ 1 holds. Thus, we have

$T_{5} \leq t_{0}+\left[V_{2}^{\alpha(1+\alpha)+(1 / p)-2}\left(t_{0}\right) \frac{\Gamma(1+\alpha) \Gamma(1-((2 p-1) / p(1+\alpha)))}{\bar{\eta} \cdot \Gamma(2+\alpha) \Gamma(1+\alpha-((2 p-1) / p(1+\alpha)))}\right]^{1 / \alpha}=\left[60.5^{0.4404} \cdot \frac{\Gamma(1.98) \Gamma(0.2424)}{1.9608 \cdot \Gamma(2.98) \Gamma(1.2224)}\right]^{1 / 0.98}=6.689$

Therefore, drive-response system (118) can achieve finite-time projective synchronization under controller (83). And the state trajectories and error curves of FMCGNNs are as shown in Figures 2-4, which demonstrate the effectiveness of Theorems 1 and 2 .

4.2. Complete Synchronization. First, we choose the projective coefficient $\lambda=1$. We can get $\Phi_{1}(s)=y_{1}(s)-$ $2 x_{1}(s)=4, \Phi_{2}(s)=y_{2}(s)-2 x_{2}(s)=-3, V_{1}\left(t_{0}\right)=7$, and $V_{2}\left(t_{0}\right)=25$.

According to Theorem 1, it can be calculated as follows:

$$
\begin{aligned}
& \varphi_{1}=k_{1}-1.1, \\
& \varphi_{2}=k_{2}-1.1 ; \\
& \delta_{1}=h_{1}-1, \\
& \delta_{2}=h_{2}-0.9 ; \\
& \eta_{1}=q_{1}-6.36, \\
& \eta_{2}=q_{2}-8.12 .
\end{aligned}
$$

And we choose $k_{1}=3.1, k_{2}=3.1, h_{1}=1, h_{2}=0.9, q_{1}=$ 7.36, and $q_{2}=9.12$ which make $\varphi=2$ and $\eta=2$.

Then, we have a finite-time fractional-order differential inequation as follows:

$$
{ }_{t_{0}}^{C} D_{t}^{\alpha} V_{1}(t) \leq-2 V_{1}(t)-2 .
$$

Based on the result in Theorem 1, drive-response system (118) could achieve synchronization in finite time. And we can calculate the setting time $T_{1}^{*}$ as follows:

$$
T_{1}^{*} \leq t_{0}+\varphi^{-1 / \alpha} \ln \frac{\varphi V_{1}\left(t_{0}\right)+\eta}{\alpha \eta} \leq 2^{-1 / 0.98} \ln \left(\frac{2 \times 7+2}{0.98 \times 2}\right)=1.0351 \text {. }
$$

To verify Remark 4, we can calculate $T_{2}$ to demonstrate that $T_{1}^{*}$ is less conservative than $T_{2}$. It follows that

$$
T_{2} \leq t_{0}+\left[\frac{V_{1}\left(t_{0}\right) \Gamma(1+\alpha)}{\eta}\right]^{1 / \alpha}=\left(\frac{7 \cdot \Gamma(1.98)}{2}\right)^{1 / \alpha}=3.5169
$$

On the contrary, we select $p=2$ and $\varepsilon_{1}=\varepsilon_{2}=1$ for Theorem 2. And it is easy to calculate

$$
\begin{aligned}
& \bar{\varphi}_{1}=1.9608\left(k_{1}-1.4\right), \\
& \bar{\varphi}_{2}=1.9608\left(k_{2}-1.85\right) ; \\
& \bar{\delta}_{1}=1.9608\left(h_{1}-0.5\right), \\
& \bar{\delta}_{2}=1.9608\left(h_{2}-0.45\right) ; \\
& \bar{\eta}_{1}=1.9608\left(q_{1}-6.36\right), \\
& \bar{\eta}_{2}=1.9608\left(q_{2}-8.12\right) .
\end{aligned}
$$

Under the controller parameter, $k_{1}=3.1, k_{2}=3.1, h_{1}=$ $1, h_{2}=0.9, q_{1}=7.36$, and $q_{2}=9.12$ which lead to $\bar{\varphi}=2.451$ and $\bar{\eta}=1.9608$.

Hence, we have another finite-time fractional-order differential inequation as follows:

$$
{ }_{t_{0}}^{C} D_{t}^{\alpha} V_{2}(t) \leq-2.451 V_{2}(t)-1.9608 V_{2}^{1 / 2}(t) \text {. }
$$

Based on the result in Theorem 2, we can estimate the setting time $T_{3}$ as follows: 


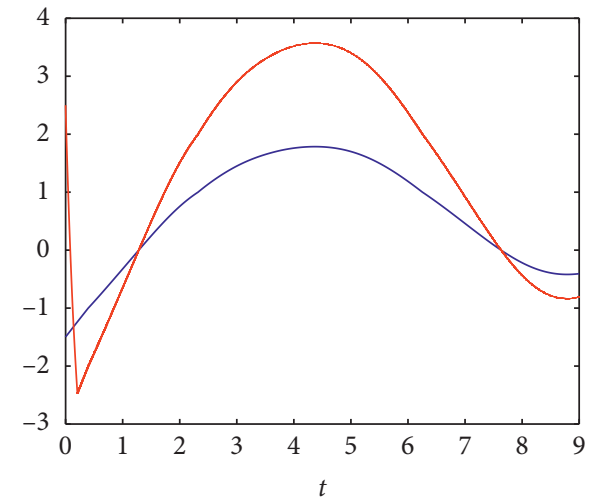

$$
-x_{1}
$$

FIGURE 2: The first dimensional state trajectories of the drive system and the response system when the projective coefficient $\lambda=2$.
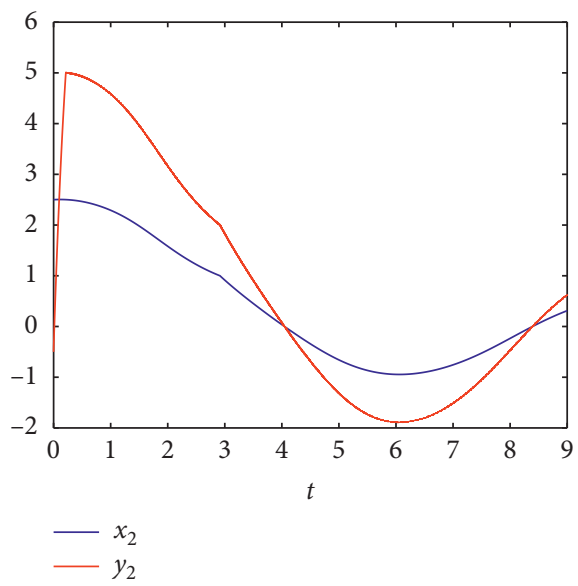

Figure 3: The second dimensional state trajectories of the drive system and the response system when the projective coefficient $\lambda=2$.

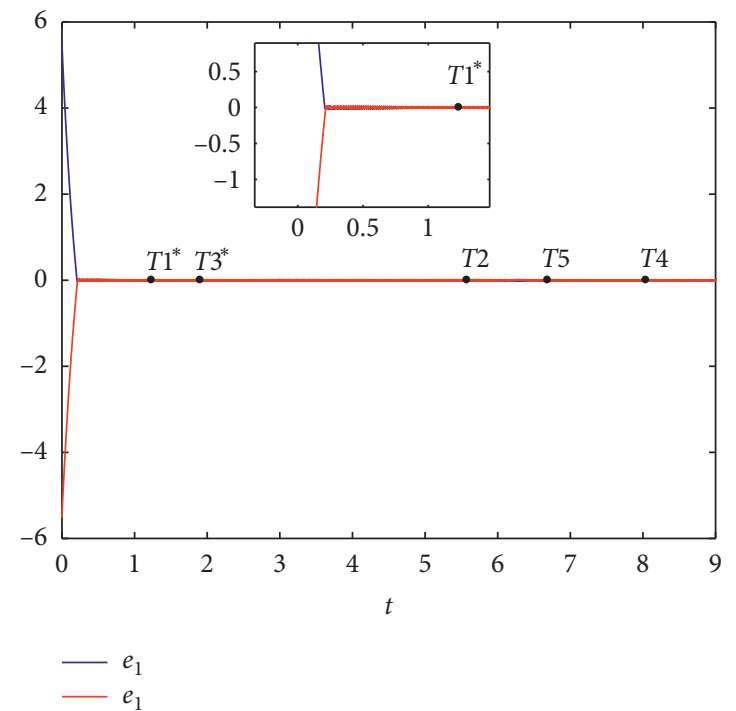

FIGURE 4: The synchronization error evolution when the projective coefficient $\lambda=2$. 


$$
T_{3}^{*} \leq t_{0}+\left[\frac{\Gamma(1+(p /(p-1))-\alpha) \bar{\varphi}}{\Gamma(1+(p /(p-1))) \Gamma(2-\alpha)}\right]^{-1 / \alpha} \ln \frac{\bar{\varphi} V_{2}^{1 / p}\left(t_{0}\right)+\bar{\eta}}{\bar{\eta} \alpha}=\left[\frac{2.451 \Gamma(2.02)}{\Gamma(3) \Gamma(1.02)}\right]^{-1 / 0.98} \ln \frac{2.451 \times \sqrt{25}+1.9608}{1.9608 \times 0.98}=1.5937
$$

To verify Remark 6, we can calculate $T_{4}$ to demonstrate that $T_{3}^{*}$ is less conservative than $T_{4}$. It follows that

$$
T_{4} \leq t_{0}+\left[V_{2}^{1 / p}\left(t_{0}\right) \frac{\Gamma(1+p) \Gamma(2-\alpha) \Gamma(1+\alpha)}{\Gamma(1+p-\alpha) \bar{\eta}}\right]^{1 / \alpha}=\left[\sqrt{25} \cdot \frac{\Gamma(3) \Gamma(1.02) \Gamma(1.98)}{1.9608 \Gamma(2.02)}\right]^{1 / 0.98}=5.1232
$$

Considering $p=2$ and $\alpha=0.98,(p-1) / p<\alpha(1+\alpha)-$ 1 holds. Thus, we have

$$
T_{5} \leq t_{0}+\left[V_{2}^{\alpha(1+\alpha)+(1 / p)-2}\left(t_{0}\right) \frac{\Gamma(1+\alpha) \Gamma(1-((2 p-1) / p(1+\alpha)))}{\bar{\eta} \cdot \Gamma(2+\alpha) \Gamma(1+\alpha-((2 p-1) / p(1+\alpha)))}\right]^{1 / \alpha}=\left[25^{0.4404} \cdot \frac{\Gamma(1.98) \Gamma(0.2424)}{1.9608 \cdot \Gamma(2.98) \Gamma(1.2224)}\right]^{1 / 0.98}=4.4965
$$

Therefore, drive-response system (118) can achieve finitetime complete synchronization under controller (83). And the state trajectories and error curves of FMCGNNs are as shown in Figures 5-7, which demonstrate the effectiveness of Theorems 1 and 2.

4.3. Antisynchronization. First, we choose the projective coefficient $\lambda=-1$. We can get $\Phi_{1}(s)=y_{1}(s)-2 x_{1}(s)=1$, $\Phi_{2}(s)=y_{2}(s)-2 x_{2}(s)=2, V_{1}\left(t_{0}\right)=3$, and $V_{2}\left(t_{0}\right)=5$.

According to Theorem 1, it can be calculated as follows:

$$
\begin{aligned}
& \varphi_{1}=k_{1}-1.1, \\
& \varphi_{2}=k_{2}-1.1 ; \\
& \delta_{1}=h_{1}-1 \\
& \delta_{2}=h_{2}-0.9 ; \\
& \eta_{1}=q_{1}-9.08, \\
& \eta_{2}=q_{2}-11.12 .
\end{aligned}
$$

And we choose $k_{1}=3.1, k_{2}=3.1, h_{1}=1, h_{2}=0.9, q_{1}=$ 10.08, and $q_{2}=12.12$ which make $\varphi=2$ and $\eta=2$.

Then, we have a finite-time fractional-order differential inequation as follows:

$$
{ }_{t_{0}}^{C} D_{t}^{\alpha} V_{1}(t) \leq-2 V_{1}(t)-2
$$

Based on the result in Theorem 1, drive-response system (118) could achieve synchronization in finite time. And we can calculate the setting time $T_{1}^{*}$ as follows:

$$
T_{1}^{*} \leq t_{0}+\varphi^{-1 / \alpha} \ln \frac{\varphi V_{1}\left(t_{0}\right)+\eta}{\alpha \eta} \leq 2^{-1 / 0.98} \ln \left(\frac{2 \times 3+2}{0.98 \times 2}\right)=0.6934
$$

To verify Remark 4, we can calculate $T_{2}$ to demonstrate that $T_{1}^{*}$ is less conservative than $T_{2}$. It follows that

$$
T_{2} \leq t_{0}+\left[\frac{V_{1}\left(t_{0}\right) \Gamma(1+\alpha)}{\eta}\right]^{1 / \alpha}=\left(\frac{3 \cdot \Gamma(1.98)}{2}\right)^{1 / \alpha}=1.4814
$$

On the contrary, we select $p=2$ and $\varepsilon_{1}=\varepsilon_{2}=1$ for Theorem 2. And it is easy to calculate

$$
\begin{aligned}
& \bar{\varphi}_{1}=1.9608\left(k_{1}-1.4\right), \bar{\varphi}_{2}=1.9608\left(k_{2}-1.85\right) \\
& \bar{\delta}_{1}=1.9608\left(h_{1}-0.5\right), \bar{\delta}_{2}=1.9608\left(h_{2}-0.45\right) ; \\
& \bar{\eta}_{1}=1.9608\left(q_{1}-9.08\right), \bar{\eta}_{2}=1.9608\left(q_{2}-11.12\right) .
\end{aligned}
$$

Under the controller parameter, $k_{1}=3.1, k_{2}=3.1, h_{1}=$ $1, h_{2}=0.9, q_{1}=10.08$, and $q_{2}=12.12$ which lead to $\bar{\varphi}=2.451$ and $\bar{\eta}=1.9608$.

Hence, we have another finite-time fractional-order differential inequation as follows:

$$
{ }_{t_{0}}^{C} D_{t}^{\alpha} V_{2}(t) \leq-2.451 V_{2}(t)-1.9608 V_{2}^{1 / 2}(t) \text {. }
$$

Based on the result in Theorem 2, we can estimate the setting time $T_{3}$ as follows: 


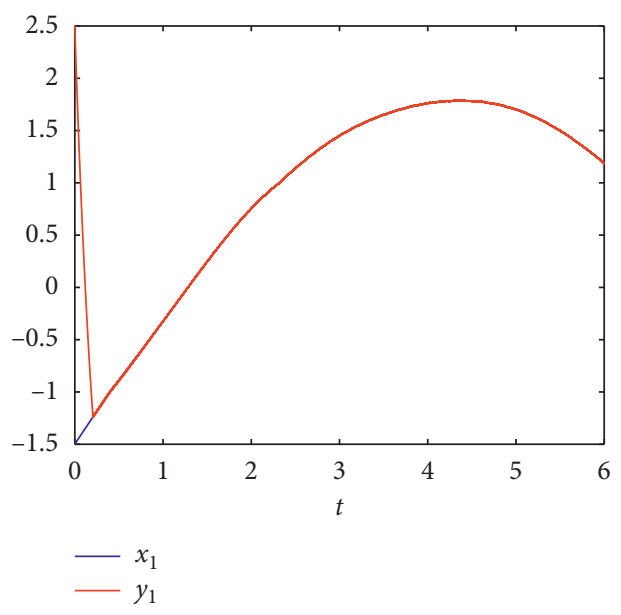

FIgURE 5: The first dimensional state trajectories of the drive system and the response system when the projective coefficient $\lambda=1$.

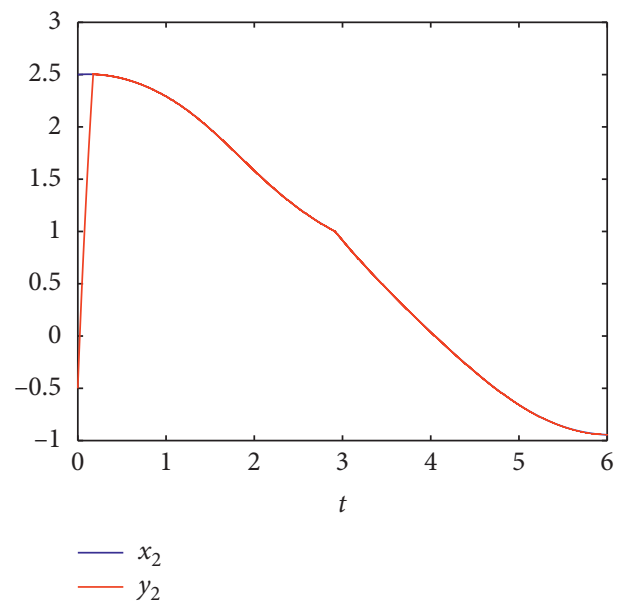

Figure 6: The second dimensional state trajectories of the drive system and the response system when the projective coefficient $\lambda=1$.

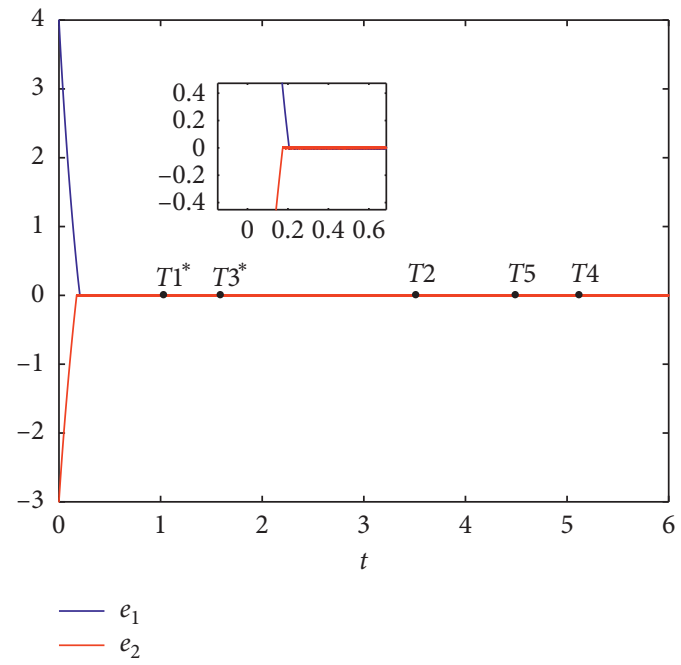

FIGURE 7: The synchronization error evolution when the projective coefficient $\lambda=1$. 


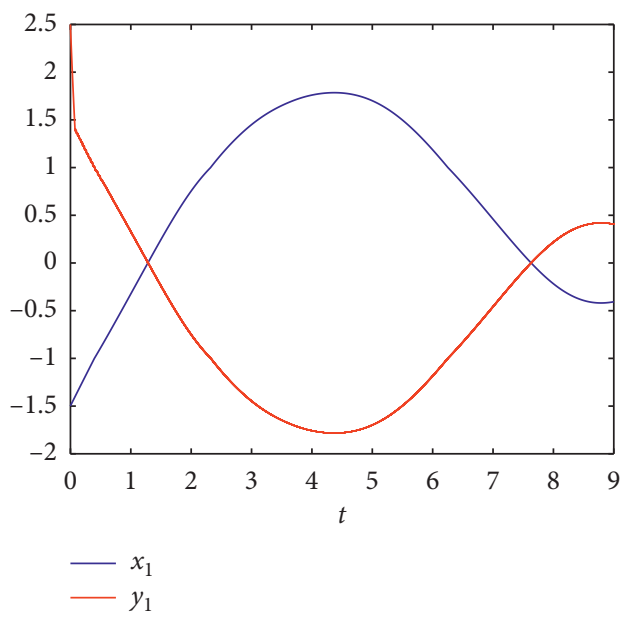

FIgURE 8: The first dimensional state trajectories of the drive system and the response system when the projective coefficient $\lambda=-1$.

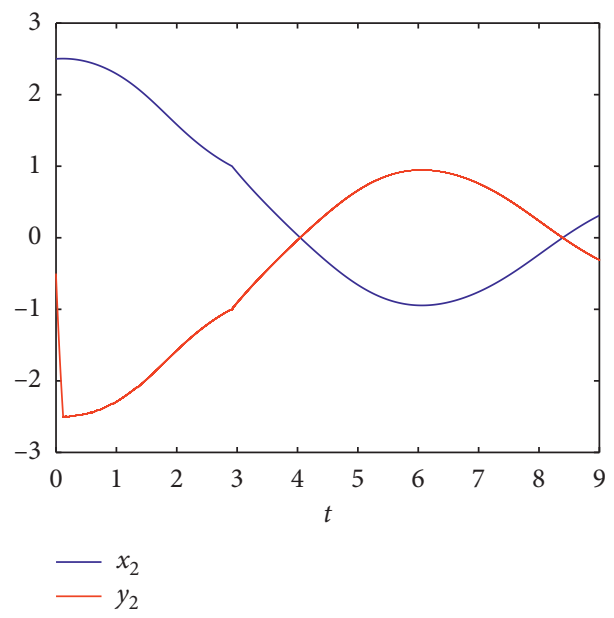

FIGURE 9: The second dimensional state trajectories of the drive system and the response system when the projective coefficient $\lambda=-1$.

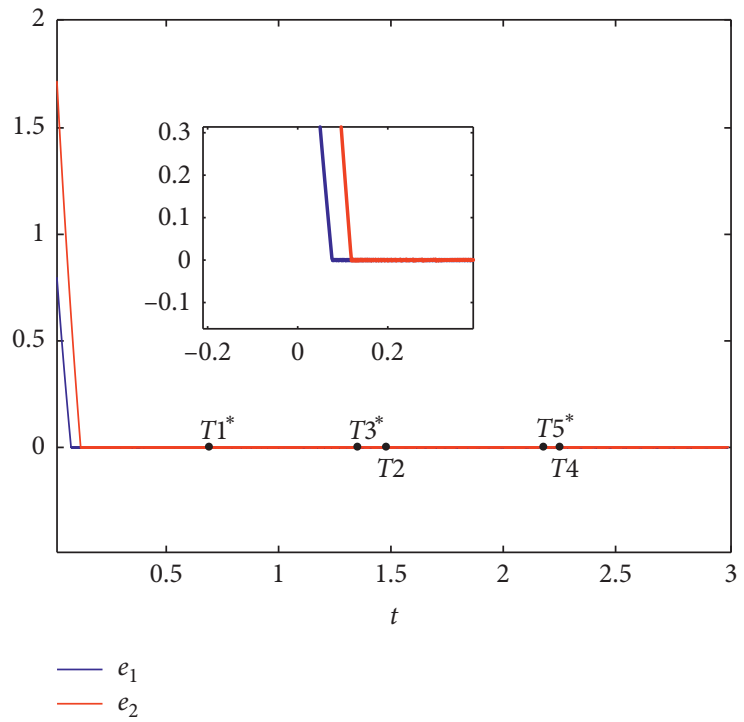

Figure 10: The synchronization error evolution when the projective coefficient $\lambda=-1$. 


$$
T_{3}^{*} \leq t_{0}+\left[\frac{\Gamma(1+(p /(p-1))-\alpha) \bar{\varphi}}{\Gamma(1+(p /(p-1))) \Gamma(2-\alpha)}\right]^{-1 / \alpha} \ln \frac{\bar{\varphi} V_{2}^{1 / p}\left(t_{0}\right)+\bar{\eta}}{\bar{\eta} \alpha}=\left[\frac{2.451 \Gamma(2.02)}{\Gamma(3) \Gamma(1.02)}\right]^{-1 / 0.98} \ln \frac{2.451 \times \sqrt{5}+1.9608}{1.9608 \times 0.98}=1.3539
$$

To verify Remark 6, we can calculate $T_{4}$ to demonstrate that $T_{3}^{*}$ is less conservative than $T_{4}$. It follows that

$$
\begin{aligned}
T_{4} & \leq t_{0}+\left[V_{2}^{1 / p}\left(t_{0}\right) \frac{\Gamma(1+p) \Gamma(2-\alpha) \Gamma(1+\alpha)}{\Gamma(1+p-\alpha) \bar{\eta}}\right]^{1 / \alpha} \\
& =\left[\sqrt{5} \cdot \frac{\Gamma(3) \Gamma(1.02) \Gamma(1.98)}{1.9608 \Gamma(2.02)}\right]^{1 / 0.98}=2.2539
\end{aligned}
$$

$$
T_{5} \leq t_{0}+\left[V_{2}^{\alpha(1+\alpha)+(1 / p)-2}\left(t_{0}\right) \frac{\Gamma(1+\alpha) \Gamma(1-(2 p-1) / p(1+\alpha))}{\bar{\eta} \cdot \Gamma(2+\alpha) \Gamma(1+\alpha-(2 p-1) / p(1+\alpha))}\right]^{1 / \alpha}=\left[5^{0.4404} \cdot \frac{\Gamma(1.98) \Gamma(0.2424)}{1.9608 \cdot \Gamma(2.98) \Gamma(1.2224)}\right]^{1 / 0.98}=2.1816
$$

Considering $p=2$ and $\alpha=0.98,((p-1) / p)<\alpha(1+\alpha)-$ 1 holds. Thus, we have
Therefore, drive-response system (118) can achieve finite-time antisynchronization under controller (83). And the state trajectories and error curves of FMCGNNs are as shown in Figures 8-10, which demonstrate the effectiveness of Theorems 1 and 2 .

\section{Conclusion}

In this paper, we have extensively discussed the finite-time projective synchronization for a class of FMNNs which are translated to a type of fractional-order systems with time delays and uncertain parameters. By means of applying the differential inequality of the Caputo derivative and the asymptotic expansion property of Mittag-Leffler function, three useful properties are proposed which play a vital role in calculating the settling time of finite-time synchronization. Besides the time-delayed feedback control utilized in this paper, other control approaches, such as impulsive control, intermittent control, and pinning control, can also be applied in the future work for studying projective synchronization of memristive neural networks with mixed timevarying delays.

\section{Data Availability}

All data included in this study are available upon request by contact with the corresponding author.

\section{Conflicts of Interest}

The authors declare that they have no conflicts of interest.

\section{Acknowledgments}

This work was supported in part by the National Natural Science Foundation of China (nos. 51407012 and 61601059), the Natural Science Foundation of Shaanxi Province
(2020JM-256), Technology Innovation Leading Program of Shaanxi (no. 2020QFY03-01), and the Fundamental Research Funds for the Central Universities, CHD (no. 300102329102).

\section{References}

[1] K. Matsuoka, M. Ohoya, and M. Kawamoto, "A neural net for blind separation of nonstationary signals," Neural Networks, vol. 8, no. 3, pp. 411-419, 1995.

[2] S. Abe, J. Kawakami, and K. Hirasawa, "Solving inequality constrained combinatorial optimization problems by the hopfield neural networks," Neural Networks, vol. 5, no. 4, pp. 663-670, 1992.

[3] C. M. Bishop, Neural Networks for Pattern Recognition, Oxford University Press, Oxford, UK, 1995.

[4] Y. V. Pershin and M. Di Ventra, "Experimental demonstration of associative memory with memristive neural networks," Neural Networks, vol. 23, no. 7, pp. 881-886, 2010.

[5] L. Chua, "Memristor-the missing circuit element," IEEE Transactions on Circuit Theory, vol. 18, no. 5, pp. 507-519, 1971.

[6] D. B. Strukov, G. S. Snider, D. R. Stewart, and R. S. Williams, "The missing memristor found," Nature, vol. 453, no. 7191, pp. 80-83, 2008.

[7] K. Mathiyalagan, J. H. Park, and R. Sakthivel, "Novel results on robust finite-time passivity for discrete-time delayed neural networks," Neurocomputing, vol. 177, pp. 585-593, 2016.

[8] K. Gopalsamy and X.-z. He, "Stability in asymmetric hopfield nets with transmission delays," Physica D: Nonlinear Phenomena, vol. 76, no. 4, pp. 344-358, 1994.

[9] H. Zhang, R. Ye, S. Liu, J. Cao, A. Alsaedi, and X. Li, "Lmibased approach to stability analysis for fractional-order neural networks with discrete and distributed delays," International Journal of Systems Science, vol. 49, no. 3, pp. 537-545, 2018.

[10] Y. Fan, X. Huang, H. Shen, and J. Cao, "Switching eventtriggered control for global stabilization of delayed memristive neural networks: an exponential attenuation scheme," Neural Networks, vol. 117, pp. 216-224, 2019. 
[11] L. Yao, Z. Wang, X. Huang, Y. Li, H. Shen, and G. Chen, "Aperiodic sampled-data control for exponential stabilization of delayed neural networks: a refined two-sided loopedfunctional approach," IEEE Transactions on Circuits and Systems II: Express Briefs, vol. 121, p. 1, 2020.

[12] H. Wang, Y. Yu, and G. Wen, "Stability analysis of fractionalorder hopfield neural networks with time delays," Neural Networks, vol. 55, pp. 98-109, 2014.

[13] W. Liu, M. Jiang, and M. Yan, "Stability analysis of memristor-based time-delay fractional-order neural networks," Neurocomputing, vol. 323, pp. 117-127, 2019.

[14] W. Zhang, H. Zhang, J. Cao, F. E. Alsaadi, and D. Chen, "Synchronization in uncertain fractional-order memristive complex-valued neural networks with multiple time delays," Neural Networks, vol. 110, pp. 186-198, 2019.

[15] Y. Fan, X. Huang, Y. Li, J. Xia, and G. Chen, "Aperiodically intermittent control for quasi-synchronization of delayed memristive neural networks: an interval matrix and matrix measure combined method," IEEE Transactions on Systems, Man, and Cybernetics: Systems, vol. 49, no. 11, pp. 2254-2265, 2019.

[16] L. M. Pecora and T. L. Carroll, "Synchronization in chaotic systems," Physical Review Letters, vol. 64, no. 8, pp. 821-824, 1990.

[17] T. Yang and L. O. Chua, "Impulsive stabilization for control and synchronization of chaotic systems: theory and application to secure communication," IEEE Transactions on Circuits and Systems I: Fundamental Theory and Applications, vol. 44, no. 10, pp. 976-988, 1997.

[18] S. Wen, Z. Zeng, T. Huang, Q. Meng, and W. Yao, "Lag synchronization of switched neural networks via neural activation function and applications in image encryption," IEEE Transactions on Neural Networks and Learning Systems, vol. 26, no. 7, pp. 1493-1502, 2015.

[19] V. Annovazzi-Lodi, S. Donati, and A. Sciré, "Synchronization of chaotic lasers by optical feedback for cryptographic applications," IEEE Journal of Quantum Electronics, vol. 33, no. 9, pp. 1449-1454, 1997.

[20] Q. Gan, R. Xu, and X. Kang, "Synchronization of chaotic neural networks with mixed time delays," Communications in Nonlinear Science and Numerical Simulation, vol. 16, no. 2, pp. 966-974, 2011.

[21] C. Huang and J. Cao, "Active control strategy for synchronization and anti-synchronization of a fractional chaotic financial system," Physica A: Statistical Mechanics and Its Applications, vol. 473, pp. 262-275, 2017.

[22] H. Zhang, X.-Y. Wang, and X.-H. Lin, "Topology identification and module-phase synchronization of neural network with time delay," IEEE Transactions on Systems, Man, and Cybernetics: Systems, vol. 47, no. 6, pp. 885-892, 2016.

[23] W. Zhang, J. Cao, R. Wu, F. E. Alsaadi, and A. Alsaedi, "Lag projective synchronization of fractional-order delayed chaotic systems," Journal of the Franklin Institute, vol. 356, no. 3, pp. 1522-1534, 2019.

[24] X. Yang, J. Cao, and W. Yu, "Exponential synchronization of memristive Cohen-Grossberg neural networks with mixed delays," Cognitive Neurodynamics, vol. 8, no. 3, pp. 239-249, 2014.

[25] S. P. Bhat and D. S. Bernstein, "Continuous finite-time stabilization of the translational and rotational double integrators," IEEE Transactions on Automatic Control, vol. 43, no. 5, pp. 678-682, 1998.

[26] X. Qin, C. Wang, L. Li, H. Peng, Y. Yang, and L. Ye, “Finitetime projective synchronization of memristor-based neural networks with leakage and time-varying delays," Physica A: Statistical Mechanics and Its Applications, vol. 531, p. 121788, 2019.

[27] M. Zheng, L. Li, H. Peng, J. Xiao, Y. Yang, and H. Zhao, "Finite-time projective synchronization of memristor-based delay fractional-order neural networks," Nonlinear Dynamics, vol. 89, no. 4, pp. 2641-2655, 2017.

[28] Y. Zhang and S. Deng, "Finite-time projective synchronization of fractional-order complex-valued memristor-based neural networks with delay," Chaos, Solitons \& Fractals, vol. 128, pp. 176-190, 2019.

[29] L. Zhang, Y. Yang, and X. Xu, "Synchronization analysis for fractional order memristive Cohen-Grossberg neural networks with state feedback and impulsive control," Physica A: Statistical Mechanics and Its Applications, vol. 506, pp. 644660, 2018.

[30] X. Peng, H. Wu, and J. Cao, "Global nonfragile synchronization in finite time for fractional-order discontinuous neural networks with nonlinear growth activations," IEEE Transactions on Neural Networks and Learning Systems, vol. 30, no. 7, pp. 2123-2137, 2019.

[31] X. Peng, H. Wu, K. Song, and J. Shi, "Global synchronization in finite time for fractional-order neural networks with discontinuous activations and time delays," Neural Networks, vol. 94, pp. 46-54, 2017.

[32] H. Wu, L. Wang, P. Niu, and Y. Wang, "Global projective synchronization in finite time of nonidentical fractional-order neural networks based on sliding mode control strategy," Neurocomputing, vol. 235, pp. 264-273, 2017.

[33] S. Yang, J. Yu, C. Hu, and H. Jiang, "Finite-time synchronization of memristive neural networks with fractional-order," IEEE Transactions on Systems, Man, and Cybernetics: Systems.

[34] R. Mainieri and J. Rehacek, "Projective synchronization in three-dimensional chaotic systems," Physical Review Letters, vol. 82 , no. 15 , pp. 3042-3045.

[35] C. Y. Chee and D. Xu, "Chaos-based m-ary digital communication technique using controlled projective synchronisation," IEE Proceedings - Circuits, Devices and Systems, vol. 153, no. 4, pp. 357-360, 2006.

[36] Y. Gu, Y. Yu, and H. Wang, "Projective synchronization for fractional-order memristor-based neural networks with time delays," Neural Computing and Applications, vol. 31, no. 10, pp. 6039-6054, 2019.

[37] H.-L. Li, C. Hu, J. Cao, H. Jiang, and A. Alsaedi, "Quasiprojective and complete synchronization of fractional-order complex-valued neural networks with time delays," Neural Networks, vol. 118, pp. 102-109, 2019.

[38] S. Yang, J. Yu, C. Hu, and H. Jiang, "Quasi-projective synchronization of fractional-order complex-valued recurrent neural networks," Neural Networks, vol. 104, pp. 104-113, 2018.

[39] G. Rajchakit, A. Pratap, R. Raja, J. Cao, J. Alzabut, and C. Huang, "Hybrid control scheme for projective lag synchronization of riemann-Liouville Sense fractional order memristive BAM NeuralNetworks with mixed delays," Mathematics, vol. 7, no. 8, p. 759, 2019.

[40] L. Zhang, Y. Yang, and F. Wang, "Projective synchronization of fractional-order memristive neural networks with switching jumps mismatch," Physica A: Statistical Mechanics and Its Applications, vol. 471, pp. 402-415, 2017.

[41] A. A. A. Kilbas, H. M. Srivastava, and J. J. Trujillo, Theory and Applications of Fractional Differential Equations, Vol. 204, Elsevier Science Limited, Berlin, Germany, 2006. 
[42] I. Podlubny, "Fractional differential equations," Mathematics in Science and Engineering, vol. 198, 1999.

[43] Z. Wong and fnm Zhao, "Exponential asymptotics of the mittag-leffler function," Constructive Approximation, vol. 18, no. 3, pp. 355-385, 2002.

[44] H. Deng and M. Krstić, "Stochastic nonlinear stabilization - I: a backstepping design," Systems \& Control Letters, vol. 32, no. 3, pp. 143-150, 1997.

[45] X. Yang and J. Cao, "Finite-time stochastic synchronization of complex networks," Applied Mathematical Modelling, vol. 34, no. 11, pp. 3631-3641, 2010.

[46] J. Chen, Z. Zeng, and P. Jiang, "Global mittag-leffler stability and synchronization of memristor-based fractional-order neural networks," Neural Networks, vol. 51, pp. 1-8, 2014.

[47] A. Pratap, R. Raja, J. Cao, C. P. Lim, and O. Bagdasar, "Stability and pinning synchronization analysis of fractional order delayed cohen-grossberg neural networks with discontinuous activations," Applied Mathematics and Computation, vol. 359, pp. 241-260, 2019.

[48] A. F. Filippov, Differential Equations with Discontinuous Righthand Sides: Control Systems, Vol. 18, Springer Science \& Business Media, Berlin, Germany, 2013.

[49] Y. S. Ledyaev, F. Clarke, P. Wolenski, and R. Stern, Nonsmooth Analysis and Control Theory, Springer-Verlag, Berlin, Germany, 1998.

[50] J. P. Aubin and A. Cellina, Differential Inclusions: Set-Valued Maps and Viability Theory, Springer, Berlin, Germany, 1984.

[51] G. Ji, C. Hu, J. Yu, and H. Jiang, "Finite-time and fixed-time synchronization of discontinuous complex networks: a unified control framework design," Journal of the Franklin Institute, vol. 355, no. 11, pp. 4665-4685, 2018. 